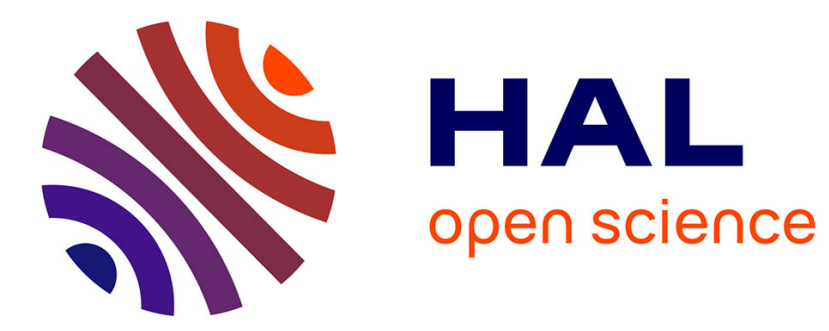

\title{
Asymmetries of the visual system and their influence on visual performance and oculomotor dynamics
}

\author{
Ómar I Jóhannesson, Jérôme Tagu, Árni Kristjánsson
}

\section{To cite this version:}

Ómar I Jóhannesson, Jérôme Tagu, Árni Kristjánsson. Asymmetries of the visual system and their influence on visual performance and oculomotor dynamics. European Journal of Neuroscience, 2018, 48 (11), pp.3426-3445. 10.1111/ejn.14225 . hal-03157767

\section{HAL Id: hal-03157767 \\ https://hal.science/hal-03157767}

Submitted on 5 May 2021

HAL is a multi-disciplinary open access archive for the deposit and dissemination of scientific research documents, whether they are published or not. The documents may come from teaching and research institutions in France or abroad, or from public or private research centers.
L'archive ouverte pluridisciplinaire HAL, est destinée au dépôt et à la diffusion de documents scientifiques de niveau recherche, publiés ou non, émanant des établissements d'enseignement et de recherche français ou étrangers, des laboratoires publics ou privés.

\section{(ㅇ)(1) $\$$}

Distributed under a Creative Commons Attribution - NonCommercial - NoDerivatives $\mid 4.0$ 


\section{Asymmetries of the visual system and their influence on visual performance and oculomotor dynamics}

Ómar I. Jóhannesson ${ }^{1, *}$, Jérôme Tagu² \& Árni Kristjánsson ${ }^{1,3}$

${ }^{1}$ Icelandic Vision Laboratory, School of Health Sciences, University of Iceland Sturlugötu 3, 101 Reykjavík Iceland

2 EA 7326 Vision Action Cognition Laboratory, Institute of Psychology, Paris Descartes University, Sorbonne-Paris-Cité, Boulogne-Billancourt, France. email: jerome.tagu@parisdescartes.fr

3 e-mail: ak@hi.is

*Corresponding author: Ómar I. Jóhannesson, e-mail: omarjo@hi.is

Running title: Nasal-temporal asymmetries in the visual system

Keywords: Nasal-temporal asymmetries, saccadic eye movements, peak velocities, visual attention, visual neuroscience

Declarations of interest: none

This article has been accepted for publication and undergone full peer review but has not been through the copyediting, typesetting, pagination and proofreading process, which may lead to differences between this version and the Version of Record. Please cite this article as:

Jóhannesson, Ó. I., Tagu, J., \& Kristjánsson, Á. (2018). Asymmetries of the visual system and their influence on visual performance and oculomotor dynamics. European Journal of Neuroscience, 48(11), 3426-3445, https://doi.org/10.1111/ejn.14225 


\begin{abstract}
Our representation of the visual field is not homogenous. There are differences in resolution not only between the fovea and regions eccentric to it, but also between the nasal and temporal hemiretinae, that can be traced to asymmetric distributions of photoreceptors and ganglion cells. We review evidence for differences in visual and attentional processing and oculomotor behaviour that can be traced to asymmetries of the visual system, mainly emphasising nasal-temporal asymmetries. Asymmetries in the visual system manifest in various measures, in basic psychophysical tests of visual performance, attentional processing, choice behaviour, saccadic peak velocity and latencies. Nasal-temporal asymmetries on saccadic latency seem primarily to occur for express saccades. Neural asymmetries between the upper and lower hemifields are strong and cause corresponding differences in performance between the hemifields. There are interesting individual differences in asymmetric processing which seem to be related to the strength of eye dominance. These neurophysiological asymmetries and the corresponding asymmetries in visual performance and oculomotor behaviour can strongly influence experimental results in vision and must be considered during experimental design and the interpretation of results.
\end{abstract}




\section{Introduction}

When sudden events in the visual field grab our attention, such attentional capture is the result of a process that rapidly converts light into neural signals. This process must be fast so that we can quickly react to danger, such as a predator, or an approaching car. There is, indeed, evidence that recognition of visual scenes is fast, and can occur as quickly as in $150 \mathrm{~ms}$ (Thorpe et al. 1996). If our attention is drawn to visual events that occur in the periphery, we typically quickly move the centre of our gaze there by making a saccade (Kristjánsson, 2011). Suddenly appearing stimuli in the periphery might be important since they may signal danger. Therefore, prioritizing processing of stimuli in the periphery might be useful from an evolutionary point of view. Neural projections from the retina to eye movement control centres in the mesencephalon are accordingly very fast, since the fastest human eye movement responses to visual events can occur in around 100 ms (Edelman et al. 2007; Edelman \& Keller, 1996; Fischer \& Boch, 1983).

In this review, our focus will be on attention shifts and eye movements to peripheral visual stimuli. Our main goal is to assess to what degree it matters where in the visual field the stimuli appear, for example on which part of the retina the stimuli are projected to. Does it matter where sudden events appear for how strongly and how quickly we react to them and shift our gaze there? While this is a simple question, it nevertheless requires a rather complicated answer, involving different levels of explanation. One part of the answer involves discussion of neurophysiology, or the basic characteristics of the neural mechanisms responsible for these eye movements. Secondly, any attempt to answer this question requires discussion of the particularities of the tests used to assess any asymmetries. This latter way of answering the question involves a more functional viewpoint.

To cut to the chase, there is indeed good evidence for asymmetrical processing within our visual system, depending on where in the visual field stimuli appear. The visual system preferentially processes stimuli within certain parts of the visual field, for example by devoting more neural machinery to those stimuli (Anderson et al. 1991; Curcio et al., 1987; Osterberg, 1935; Perry et al. 1984; Silva et al., 2018; see further discussion below). Asymmetries in the visual system are interesting from a number of perspectives. They are important for understanding vision not only from a 
neurophysiological viewpoint, but also for understanding both attentional orienting within the visual field and eye movements, since attention and eye movements are tightly coupled (Deubel \& Schneider, 1996; Kowler et al., 1995; see e.g. Kristjánsson, 2011 for review). We discuss whether there may be any benefits to a particular organization that causes such asymmetries. Such asymmetries may in some cases serve a function, while in others the asymmetries may have developed as by-products of other functions.

\subsection{Definitions}

Before proceeding further, some basic definitions should be made. Figure 1 shows the neural projections from the retina to the main brain regions involved in vision and eye movement generation and how the visual fields are projected onto the retina. Figure 2 then shows how different saccade types are defined with respect to the stimulation sites and saccade projections. Abducting/adducting and centripetal/centrifugal saccades, all have fixed reference points with respect to the body. Centripetal saccades are made from the periphery of the orbit to its centre (the primary position of the eyeball) and centrifugal saccades are made from the centre into the periphery. Note that S1 and S2 of Figure 2 are so-called symmetric saccades (Becker, 1989) as they include both a centripetal and a centrifugal component. Adducting saccades are directed from the periphery of body towards its centre line. For abducting saccades, this is reversed. Because the nasal and temporal sides are defined with respect to the visual fields (see Figures 1 and 2) the position of the eyeball within the orbit does not affect what is nasal and what is temporal. The left part of the visual field of the right eye is its nasal hemifield and projects to the temporal hemiretina and the right part of the right eye's visual field is its temporal hemifield and projects to the nasal hemiretina. The only difference between adducting/abducting and nasal/temporal saccades is therefore in how they are defined; the former are defined with respect to the anterior-posterior centre line of the body, while the latter are defined with respect to the visual hemifields. All adducting saccades are nasal saccades and all abducting saccades are temporal saccades.

There are three commonly used methods used for eliciting saccades: the gap (the fixation point disappears prior to the onset of the target), the step (the offset of the fixation point and the onset of the target happen simultaneously) and the overlap paradigms (the offset of the fixation point happens after the onset of the target). 
Saccadic latency has been found to be shorter in the gap paradigm (about $150 \mathrm{~ms}$ or less) than in the step and overlap paradigms. In the step paradigm, the latency is typically around $200 \mathrm{~ms}$ and in the overlap paradigm the latency in commonly observed to be about $250 \mathrm{~ms}$ or longer (e.g. Saslow 1967).

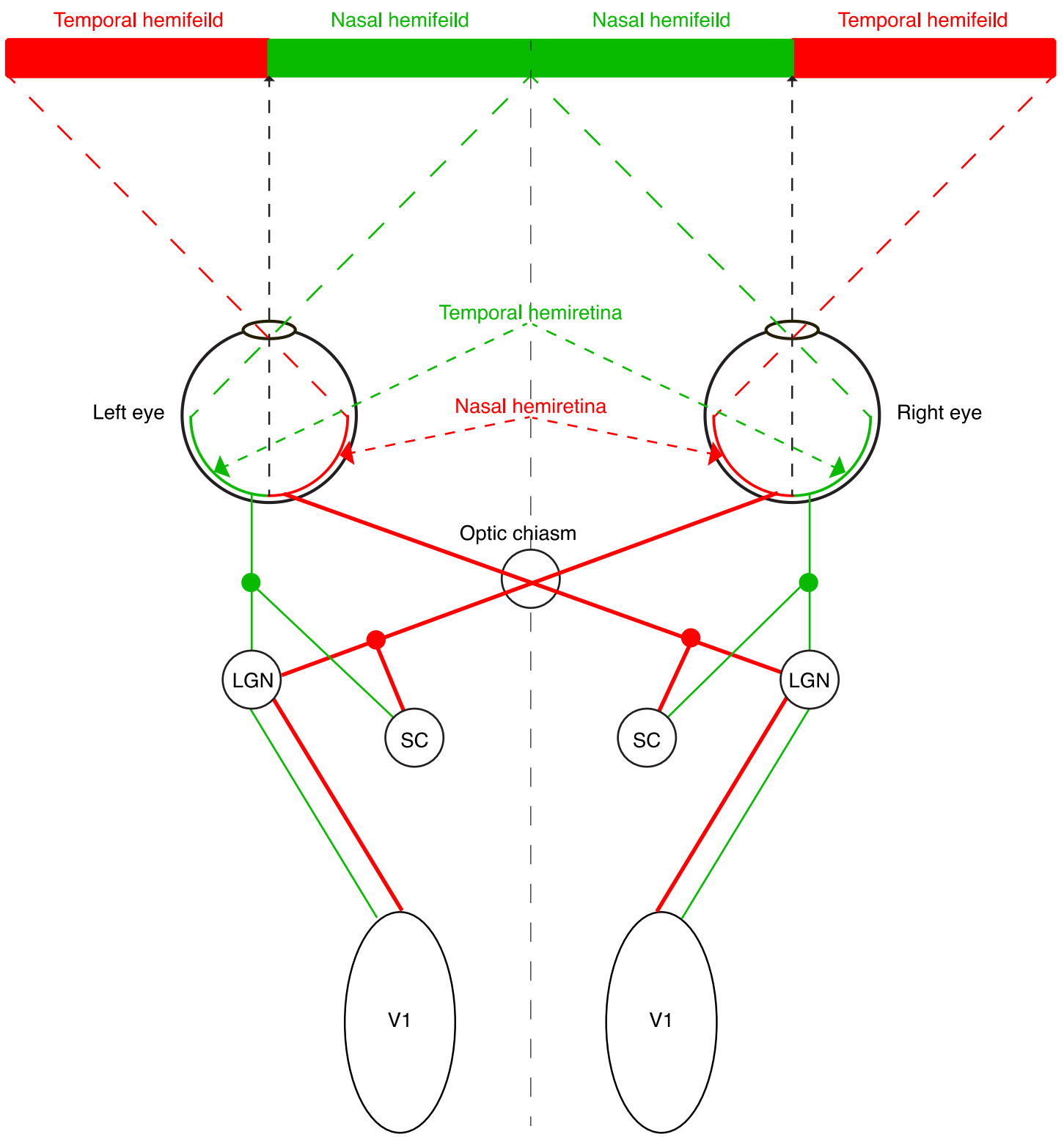

Figure 1. The visual pathways. The figure shows the projections from the nasal (red lines) and the temporal (green lines) hemifields through the nasal and temporal hemiretinae, to the SC (the retinotectal pathway), and through the LGN to V1 (thalamocortical pathway). The neurophysiological nasal-temporal asymmetry (NTA) is reflected by wider red than green lines. (Abbreviations: SC: Superior colliculi; LGN: Lateral geniculate nucleus; V1: primary visual cortex).

Saccadic eye movements can involve different cortical and subcortical networks according to the situation triggering the saccade. Theoretically, saccades can be defined 
as reflexive and voluntary saccades (e.g. Walker et al. 2000a). Reflexive saccades are triggered by sudden events appearing in the environment and are fast and automatic (these are for example the saccades you make when danger approaches your car while you are driving on the highway). Under laboratory settings, the target usually appears at random locations after a random time interval. But in contrast to the unexpected appearance of a "target" in natural settings the appearance of a target in an experiment is not completely unexpected; the participant knows that a target will appear but does not know exactly where and when. This means that reflexive saccades under laboratory settings might, at least to some extent, be influenced by top-down factors. Express saccades - a subclass of reflexive saccades - have very short latencies $(<120-130 \mathrm{~ms}$; Delinte et al. 2002; Fischer \& Ramsperger 1984; Heeman et al. 2017) and have been thought to be the fastest possible saccade type and to reflect a visuomotor "grasp" reflex (e.g. Fischer and Ramsperger 1984), partly driven by the subcortical retinotectal pathway (Dorris et al. 1997; Hall \& Lee, 1997; see also Koller \& Rafal, 2018). Express saccades occur most often in the gap paradigm. The other "regular" reflexive saccades are thought to be driven by the thalamocortical pathway, which projects to the parietal eye field and then to the superior colliculus (SC) (Pierrot-Deseilligny et al., 1995; White \& Munoz, 2011). Note that whether express saccades can be considered a distinct class of saccades has been hotly debated (Fischer \& Weber, 1993; Heeman, et al., 2017; Jüttner and Wolf 1992; Kingstone and Klein, 1993; Wenban-Smith and Findlay, 1991). Voluntary saccades, on the other hand, are strongly mediated by top-down influences. These saccades are those we make during reading or while looking for a friend in a crowded square. They are driven by the thalamocortical pathway which projects to the parietal eye field and frontal eye field, and then to the SC (Pierrot-Deseilligny et al., 1995; White \& Munoz, 2011). The signature of these saccades is a longer and more variable latency. The presence of nasal-temporal asymmetries (NTAs) in these different saccade types has been examined with the gap (e.g. Bibi \& Edelman, 2009; Jóhannesson et al. 2018), step (e.g. Jóhannesson et al. 2012; Tagu et al. 2018a) and overlap paradigms (e.g. Knox \& Wolohan, 2015; Knox et al. 2017; Tagu et al., 2018a). 


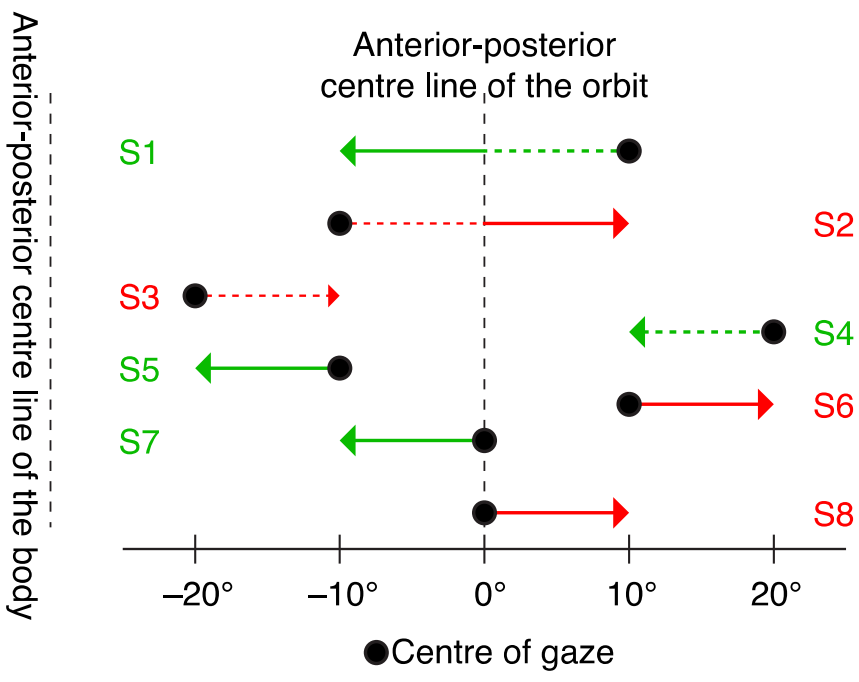

Figure 2. Saccadic movements types of the right eye. Adducting (S1, S4, S5 and S7) and abducting (S2, S3, S6 and S8) saccades are defined with respect to the anterior-posterior centre line of the body. Centripetal (S3 and S4) and centrifugal (S5, S6, S7 and S8) saccades are defined with respect to the anteriorposterior centre line of the orbit. Nasal (S1, S4, S5 and S7) and temporal (S2, S3, S6 and S8) saccades are defined with respect to the centre of gaze. Dotted lines represent centripetal saccades and whole lines represent centrifugal saccades. Red lines represent saccades (abducting) into the temporal hemifield and green lines represent saccades (adducting) into the nasal hemifield.

\subsection{Overview}

This review is divided into two main sections: In Section 2 we discuss NTAs in the visual systems, followed by discussion of other asymmetries in the visual system (Section 3). In Section 2.1 we start by reviewing evidence showing biological (or neurophysiological) NTAs in the retina and the optic nerve. NTAs in the development of the visual system are discussed in Section 2.2. In Section 2.3 we discuss NTAs in visual sensitivity, acuity and resolution. We then discuss NTAs in visual attention in section 2.4 and in Section 2.5 we review evidence concerning NTAs in saccades. While the previous sections review NTAs in studies involving monocular viewing conditions, the Section 3.1 is devoted to studies of NTAs during binocular viewing. We then discuss evidence regarding other visual asymmetries, such as vertical asymmetries in eye movements and attention (Section 3.2) and asymmetries between centrifugal and centripetal saccades (Section 3.3). Finally, we discuss individual variability in NTAs in Section 4. 


\section{Nasal-temporal asymmetries}

When studying NTAs - whether in oculomotor behaviour or visual performance - the view of one eye is typically blocked, since what is nasal for one eye is temporal for the other (see Figure 1). The SC, a key area in the mesencephalon for eye movement control, contains a retinotopic map (White \& Munoz, 2011), where a substantially bigger area is devoted to the foveal retina than the peripheral retina (White \& Munoz, 2011). Under monocular viewing the right visual field of the right eye (the temporal hemifield) is projected to the nasal hemiretina and its left visual field (nasal hemifield) is projected to its temporal hemiretina (Figure 1). During binocular viewing, the signals from the temporal hemiretina of one eye and signals from the nasal hemiretina of the other are combined in the retinotopic map in SC and - at least to some extent - in the optic nerve, counteracting effects of biological NTAs.

\section{$2.1 \quad$ Neurophysiological asymmetries}

Photoreceptors in the primate retina turn light into neural messages that are then conveyed to visual processing centres in the cerebral cortices and the midbrain. The distribution of these receptors is not uniform. Firstly, there is good evidence that visual receptor density decreases the further away from the fovea we go (Anderson et al. 1991; Osterberg, 1935). This is also true for ganglion cell density (Perry et al. 1984). This causes corresponding decreases in visual resolution in the periphery (e.g. Anderson et al., 1991; Anstis, 1974; Thibos et al. 1987). But more importantly for our purposes, there are asymmetries in the distribution of cells in the nasal and temporal parts of the retina: Perry and Cowey (1985) found that at peripheral locations, cone density was higher in the nasal- than in the temporal hemiretina of each eye. Curcio et al. (1987) then confirmed this finding for monkeys and humans.

Importantly, this asymmetry in cone density is associated with an asymmetry in ganglion cell density in primates, with higher density in the nasal than the temporal part of the retina (Curcio \& Allen 1990; Rolls \& Cowey 1970; Whitteridge, 1965). This NTA is particularly prominent at higher eccentricities, which is important for the current question of attentional and oculomotor responses to peripheral events. Perry and Cowey (1985) found that the number of ganglion cells in the retinae of their monkeys varied from $1.4 \times 10^{6}$ to $1.8 \times 10^{6}$. At about $0.5 \mathrm{~mm}$ from the fovea the 
ganglion cell density peaked with higher density in the nasal than in the temporal part of the retina. This asymmetry was considerably higher in the periphery where the density in the temporal hemiretina dropped to half the nasal hemiretina density.

Additionally, the ratio of cones versus ganglion cells is not uniform across the retina, since in the eyes of Macaca fascicularis, the ganglion cell to receptor ratios change from 4 to 1 at centre to $1: 1$ about $15^{\circ}$ away from the fovea (Wässle et al., 1989, 1990). Within $3^{\circ}$ temporally and $4^{\circ}$ nasally there are more than 2 ganglion cells per cone but at $14^{\circ}$ temporally and $20^{\circ}$ nasally the ratio is about $1: 1$. Further out in the periphery, cones are more numerous than ganglion cells. Furthermore, Wässle et al. (1990) found that the density of ganglion cells decreased faster with eccentricity for the temporal than the nasal hemiretina. If there are fewer ganglion cells per cone in the temporal than nasal hemiretina this may lead to correspondingly lower spatial acuity. Overall, the density of both cones and ganglion cells is higher in the nasal than temporal hemiretina at the same eccentricity (e.g. Curcio \& Allen 1990).

The axons of the ganglion cells from each eye are then grouped to form the optic nerves and the NTAs in ganglion cell density produce NTAs in the optic nerves. Indeed, $60 \%$ of the axons in each optic nerve project from nasal ganglion cells while the remaining 40\% project from temporal ones (Rachel \& Reese, 2009; Williams et al. 1995). These NTAs in the optic nerves have consequences at all stages of the visual pathways, as we will discuss.

While the main visual pathway in vertebrates, other than mammals, runs from the retina to the midbrain (the retinotectal pathway), the main visual pathway for mammals runs from the retina to the cortex - the thalamocortical pathway. In mammals, only about $10 \%$ of the optic nerve projects from the eye to the $\mathrm{SC}$ to form the retinotectal pathway, while about $90 \%$ project to the lateral geniculate nucleus (LGN) and then to the visual cortex to form the thalamocortical pathway (Perry et al. 1984). Note that in the thalamocortical pathway, the visual cortex relays information to the SC (both directly and indirectly; see White \& Munoz, 2011 for review). This is important as the SC play a crucial role in generating eye movements. There is evidence that the $\mathrm{SC}$ receive more direct input from the nasal than the temporal hemiretina, which suggests that NTAs occur in the retinotectal pathway. Neurophysiological studies on cats (Hubel, LeVay, \& Wiesel 1975; Sterling 1973) have revealed NTAs in projections from the hemiretinae to the SC, findings that have then been replicated in both old 
world (Itaya \& Van Hoesen 1983) and new world monkeys (Tigges \& Tigges 1981). Furthermore, Sylvester et al. (2007) found that fMRI responses to contrast reversing checkerboards were stronger for temporal hemifield stimuli (that project to the nasal hemiretina) than nasal hemifield stimuli (that project to the temporal hemiretina). Notably, this NTA in the blood-oxygen-level dependent (BOLD) signal was only observed in the SC, not the LGN nor the visual cortex. This argues for a unique asymmetry in the retinotectal projections that does not apply to the other pathways.

Williams et al., (1995) found that the ratio between nasal and temporal projection to the SC was on average 1.57 favouring the nasal hemiretina in rhesus monkeys (Macaca mulatta). Importantly, Williams et al. (1995) found similar asymmetries in projections to the LGN where the same ratio was 1.54 favouring the nasal hemiretina. In contrast to Sylvester et al. (2007), Williams et al. (1995) concluded that there were NTAs in both retinotectal and thalamocortical pathways. Note that NTAs in the LGN of macaque monkeys had already been found by Connoly and Van Essen (1984). The idea of NTAs in the thalamocortical pathway has been reinforced by findings in both monkeys (LeVay et al., 1985; Tychsen \& Burkhalter, 1997) and humans (Toosy et al., 2001) arguing for NTAs in the primary visual cortex (V1). Indeed, Tychsen and Burkhalter (1997) found that ocular dominance columns in V1 formed by the input of the nasal hemiretina were larger than those formed by information from the temporal hemiretina.

Altogether, there is strong evidence for neurophysiological NTAs, both in the retinotectal- and thalamocortical pathways. These neurophysiological NTAs are likely to strongly influence the processing of visual information by both subcortical and cortical structures.

\subsection{Nasal-temporal asymmetries during development}

A highly pertinent question in relation to NTAs of the visual system is how such asymmetries develop. Research suggests that newborns have strong NTAs, favouring the temporal hemifield, that become weaker over the course of development. Rothbart et al. (1990) argued for faster development of neural mechanisms in the infant nasal hemiretina than the temporal hemiretina. 
Lewis and Maurer (1992) used visual perimetry measurements to assess the development of the functional visual field of children from birth to 6 month old, comparing their performance with adult performance. One half of each group had their left eye patched and the other their right eye. On half the trials, two light spots of different sizes $\left(3^{\circ}\right.$ and $6^{\circ}$ ) were flashed in $15^{\circ}$ steps from the centre to $120^{\circ}$ in the nasal and temporal visual hemifields. In the other half, no lights were flashed and those trials were used as baseline. Lewis and Maurer found that with a stimulus of $3^{\circ}$, four week old infants did not orient more often to the stimulus than to a blank area in the periphery. But with increased age, the infants looked further out into the periphery and significantly longer into the temporal visual hemifield (processed by the nasal hemiretina) than into the nasal hemifield (processed by the temporal hemiretina). For example, 4 month old infants continued to orient towards a $3^{\circ}$ stimulus until eccentricities as high as $75^{\circ}$ were reached in the temporal visual hemifield while in the nasal hemifield, orientation towards the $3^{\circ}$ stimulus was only found until eccentricities of $30^{\circ}$ were reached. With a larger stimulus $\left(6^{\circ}\right)$ they oriented towards stimuli located at eccentricities reaching $105^{\circ}$ and $45^{\circ}$ in the temporal and nasal visual hemifields, respectively. Interestingly, the development of the temporal hemiretina seemed to lag behind the nasal hemiretina since this asymmetry was affected by age.

This asymmetry in infants also shows up in how they orient to facelike stimuli. Simion et al. (1998) tested how newborns, with one eye closed with a patch, oriented to three dots arranged in a facelike arrangement that conveyed eyes and mouth, compared to non-facelike stimuli (the same dots presented upside down). They found that newborns oriented preferentially to the facelike arrangement of the dots, but interestingly, this asymmetry only occurred for the temporal hemifield. Infants do indeed seem to prefer looking into their temporal visual hemifields. Johnson et al. (2000) presented upright and inverted facelike stimuli in the nasal and temporal hemifields. The infants looked more frequently (although not significantly) at facelike stimuli in the temporal than the nasal hemifield. But when inverted faces and stripes that looked similar to the inverted faces were presented, the main effect of visual hemifield was significant and the infants looked more often at stimuli in the temporal than the nasal hemifield.

Previously, Simion et al. (1995) had shown that inhibition of return was stronger for the temporal than nasal hemifields in newborns. From such findings, Johnson (2005) argued that these looking preferences and processing asymmetries of newborns reflect 
the operation of a fast, low-spatial-frequency, subcortical face-detection pathway (Vuilleumier \& Scwartz, 2001). Note that Tomalski et al. (2009) showed that this asymmetry in orienting to faces persists into adulthood, but the effect was not as large for adults as for the children.

Lewis et al. (1985) observed converging results for the ability of 1 to 2 month old infants to detect lines of varying width (by shifting gaze towards them). Their findings showed that one month old infants were able to detect $1.5^{\circ}$ wide lines at $30^{\circ}$ in the temporal visual field but not $12.8^{\circ}$ wide lines at $20^{\circ}$ in the nasal visual field. This suggests that the infants were over eight times less sensitive to stimuli at $20^{\circ}$ of eccentricity in the temporal part of the retina than at $30^{\circ}$ in the nasal part.

What holds for line detection might not hold for detecting intensity differences. In Bowering et al. (1993) the task was to detect a light of varying intensity $\left(0.43^{\circ}\right.$ in diameter) presented for $100 \mathrm{~ms}$ either $20^{\circ}$ nasally or $30^{\circ}$ temporally. They found that the sensitivity of 7 to 9 year old children was higher at $20^{\circ}$ of eccentricity in the nasal hemifield than at $30^{\circ}$ in the temporal hemifield. Bowering et al., (1993) found the same pattern for their adult participants, suggesting that this advantage of the nasal visual hemifield (temporal hemiretina) emerges early in life and does not disappear in adulthood. When considering these results, it is important to note that Vernier acuity ${ }^{1}$ is similar for both hemifields only for the first $5^{\circ}$ away from the fovea, but after that there is a clear NTA, at least for adults (Fahle \& Schmid, 1988). For example, at $20^{\circ}$ away from the fovea, Vernier acuity is approximately $70 \%$ higher in the nasal hemiretina than the temporal hemiretina.

Finally, a study of children with unilateral congenital cataracts (Bowering et al. 1993) shows that the nasal visual hemifield is affected more than the temporal visual hemifield by visual deprivation, most likely stemming from interocular competition during development and that this can have large effects upon vision later in life.

All-in-all, this evidence suggests that people are born with a strong asymmetry favouring the temporal hemifield, but also that this asymmetry decreases with age, but probably never fully disappears.

\footnotetext{
${ }^{1}$ Vernier acuity is the ability to detect misalignment between two lines where one is at the end of the other and both have the same orientation.
} 


\subsection{Neurophysiological asymmetries are reflected in visual performance}

We next turn to the question of whether the physiological asymmetries discussed above are reflected in visual perception and performance - such as visual acuity and visual sensitivity. Consistent with asymmetries in neural density and neural projections, various measures of visual perception show that corresponding asymmetries are observed in visual performance. We first discuss effects on fundamental psychophysical measures of vision and then turn to findings on visual attention.

\subsubsection{Measures of visual sensitivity, acuity and resolution}

Sutter and Tran (1992) used electroretinogram (ERG) measures to assess the photonic luminance response of retinal cells, finding higher response densities in the temporal visual hemifield (projecting to the nasal hemiretina), particularly when stimulating at locations eccentric to the blind spot. In general, the ERG amplitude as a function of eccentricity decreased by $r^{-2 / 3}(r=$ eccentricity). The main conclusion of Sutter and Tran (1992) was that the known anatomical asymmetries induced asymmetries in the photonic luminance response. Anderson et al. (1992) attempted to find a behavioural analogue of NTAs in ganglion cell density (Curcio \& Allen, 1990). They used an interferometer which minimizes the defocusing effects of the optical properties of the eye. They found that at $20^{\circ}$ and $25^{\circ}$ away from the fovea the spatial frequency limit was higher for the nasal than the temporal hemiretina (i.e., for the temporal than the nasal visual hemifield), arguing that peripheral resolution reflects the asymmetrical spacing of ganglion cells.

Anderson et al. (1991) examined whether optical, receptoral or higher-order neural properties impose limits on visual acuity or visual spatial resolution, focusing in particular on the peripheral regions of the visual field. They measured spatial contrast sensitivity functions for different parts of the retina, finding an NTA in sensitivity that corresponded roughly to the Nyquist limits ${ }^{2}$ imposed by ganglion cell density. They also found a clear NTA in contrast sensitivity, that once again accorded well with ganglion cell density. No NTAs in achromatic contrast sensitivity were found at $8^{\circ}$. However, at higher eccentricities $\left(25^{\circ}, 40^{\circ}\right.$ and $\left.55^{\circ}\right)$ NTAs increased, favouring the

\footnotetext{
${ }^{2}$ The distance between the photoreceptors in the retina limits the minimal visual acuity, i.e. stimuli or changes in a stimulus smaller than the distance between the photoreceptors cannot be detected.
} 
temporal hemifield. Chromatic contrast sensitivity was higher for the temporal than the nasal hemiretina until further than $8^{\circ}$ from the fovea where it became higher for the nasal than the temporal hemiretina.

Rovamo and Virsu (1979) argued that the functional and structural properties of the visual system show a very tight correspondence and that this is true over the whole retina. They argued, for example, that visual acuity has the same relation to the density of sampling, as seen for ganglion cells, over the whole visual field. In other words, they assumed that performance on psychophysical visual measures shows asymmetries that strongly correspond to the physiological findings. In support, Rovamo and Virsu (1979) showed that contrast sensitivity at $15^{\circ}$ in the nasal hemifield is similar to the contrast sensitivity at $30^{\circ}$ in the temporal hemifield. As discussed, cone and ganglion cell density decreases with increased eccentricity, and to a larger degree in the temporal than nasal hemiretina (e.g. Curcio et al., 1987). Note that Rovamo and Virsu (1979) only examined the left visual hemifield (measuring the nasal hemifield of the right eye and the temporal hemifield of the left eye). Silva et al. (2008) found higher contrast sensitivity performance for stimuli projected to the nasal than temporal hemiretina for both eyes. Keltgen and Swanson (2012), using Gabor sinusoids of various spatial frequencies, found a performance benefit for the nasal hemiretina and Thibos et al. (1987) found NTAs in grating discrimination (see also Fahle \& Schmid, 1988 and Paradiso \& Carney, 1988 for converging findings).

Grigsby and Tsou (1994) investigated spatial frequency sensitivity (ranging from 0.4 to 16 cycles per degree) and flicker discrimination performance (flicker range: 1.0 to $40.0 \mathrm{~Hz}$ ) as a function of eccentricity during binocular and monocular presentation. During monocular presentation, sensitivity to spatial frequency was higher in the nasal hemiretina (temporal hemifield) than in the temporal hemiretina (nasal hemifield) at $8^{\circ}$ away from the fovea and outwards. At $24^{\circ}$ and $32^{\circ}$ the spatial frequency sensitivity of the nasal hemiretina was similar to the measured binocular sensitivity but the sensitivity of the temporal hemiretina was lower than of the nasal hemiretina and lower than for binocular presentation. For flicker discrimination, no significant differences between the nasal and temporal hemiretinae were found at $4^{\circ}$ and $8^{\circ}$ but at $24^{\circ}$ and $32^{\circ}$ the sensitivity of the nasal hemiretina was higher than of the 
temporal hemiretina. Binocular flicker discrimination performance was better than monocular performance under all conditions.

An interesting perspective comes from the results of Fahle (1987) who assessed the amount of binocular inhibition over different parts of the retina using binocular rivalry. While the ratio of dominance period of the right eye divided by the dominance period for the left was roughly 1 in the fovea, it was 0.6 in the left visual field, reflecting the dominance of the left eye's temporal hemifield over the right eye's nasal hemifield. Moreover, this ratio was around 2 in the right visual field, reflecting the corresponding dominance of the right eye's temporal hemifield over the left eye's nasal hemifield.

In sum, the evidence reviewed above shows that neurophysiological findings on asymmetries in neural mechanisms are well reflected in measures of visual performance such as contrast sensitivity, Vernier acuity, and binocular inhibition. In the next section, we turn to the question of whether NTAs in the retinotectal and thalamocortical pathways induce NTAs in higher order visual processes such as visual attention.

\section{$2.4 \quad$ Nasal-temporal asymmetries in visual attention}

Above, we discussed NTAs in basic visual psychophysical measures favouring the nasal hemiretina. But many studies show that visual attention is crucial for conscious visual perception. If attention is not applied to the stimuli in the visual field, observers perform more poorly on various basic performance measures (Carrasco et al., 2004a; Kristjánsson \& Nakayama, 2003; Posner \& Cohen, 1980; Kristjánsson \& Sigurdardottir, 2008). It is therefore also important to assess whether there are NTAs in visual attention performance. One reason to expect them are the NTAs in retinotectal projections to the $\mathrm{SC}$, since the SC play a key role in attentional orienting (for a review, see e.g. Knudsen, 2011; Krauzlis et al. 2013). To investigate potential asymmetries of attentional orienting, one research line has involved testing performance with traditional measures of visual attention in healthy populations under monocular viewing to isolate nasal and temporal hemifield processing, while another line involves studies of patients with damage to certain parts of either the visual system or attentional networks.

When the visual cortex in one hemisphere is damaged, humans suffer from hemianopia, where they have no conscious vision in the hemifield projecting to the 
damaged site. But some studies have shown that people with hemianopia can nevertheless use visual information presented in their 'blind' hemifield for certain actions, a phenomenon called blindsight (Weiskrantz, 1996; Goodale \& Milner, 1992). Dodds et al (2002) tested hemianopic patients who had limited vision in one visual hemifield due to cortical damage. Their performance was at chance for forced-choice localization in the nasal hemianopic visual hemifield, while for the temporal hemianopic visual hemifield performance was intact. This finding suggested that retinotectal projections (that are stronger from the nasal than temporal hemiretina, as explained above) helped participants perform the task. A related finding is that distractor signals presented to the blind hemifield of people with hemianopia slowed saccades into their intact visual hemifield (Rafal et al., 1990), but importantly this only applied to their temporal hemifield, again arguing that the attentional grab effect is stronger for the nasal hemiretina. The important point for the current purposes is that these particular blindsight phenomena occur in the temporal hemifield that projects to the nasal hemiretina but not for the nasal hemifield, projecting to the temporal part of each retina. A likely explanation for this could be the larger neural machinery available in the nasal hemiretina, and stronger projections towards the SC from the nasal than the temporal hemiretina. It is important to note however, that Walker et al., (2000b) did not observe a similar NTA. They tested the effect of flickering and static distractors on saccadic latency in hemianopic and healthy participants under monocular viewing. The distractors were always presented in the blind hemifield of the hemianopic participants. For the healthy participants, the inhibitory effect of the distractors was stronger for the temporal visual hemifield than the nasal visual hemifield but for the hemianopic participants no such effect was found. Because the only visual pathway which is impaired in hemianopic patients is the thalamocortical one, the NTAs found for the healthy participants and not the hemianopic patients reinforce the idea that the thalamocortical pathway also involves NTAs.

But these studies also highlight a fundamental property of behavioural NTAs: their individual variability. The patients in Dodds et al. (2002) and Rafal et al. (1990) may have had NTAs in the retinotectal pathway while those in Walker et al. (2000b) did not. This individual variability in NTAs will be discussed in the Section 4 of this review. 
Other studies of attentional NTAs have involved testing of non-clinical populations, where the effects of stimulation in either hemifield upon attentional performance have been investigated. An example of such a study was reported by Posner and Cohen (1980) who asked their observers to choose whether to saccade into the nasal or temporal visual hemifield (a saccadic choice task) with one of their eyes covered. Potential targets were presented simultaneously in both hemifields. Interestingly, observers showed a clear preference for making saccades into the temporal hemifield $(79 \%$ and $21 \%$ into the temporal and nasal visual fields, respectively), and this was taken as indication that stronger retinotectal projections from the nasal than the temporal hemiretina caused this asymmetrical saccade choice. However, Bompas and Sumner (2008) reported that there was an NTA in saccadic choice even for so-called s-cone stimuli that are thought to be invisible to the retinotectal pathway and therefore not directly projected to the SC, which argues that the NTA in choice may not be unique to the retinotectal pathway, which is consistent with the physiological results of Williams et al. (1995).

Levy-Schoen (1969) was the first to notice an increase of 20 to 40 milliseconds in saccade latency when a distractor in the opposite visual field was presented along with the saccade target. Findlay (1982) and Walker et al. (1997) have also shown that when a stimulus is presented on the opposite side to a target during a saccade task, saccadic response times are longer than otherwise. Some researchers have used such findings to investigate the effects of an irrelevant distractor presented in the nasal or temporal hemifield. Rafal et al. (1991) measured the effects of such cues on attentional orienting of subjects who had one eye closed with an eye patch and found that cueing effects were stronger in the temporal than the nasal hemifield. Additionally, Rafal et al. (1989) reported NTAs for the inhibition of return effect. Inhibition of return to the previous cue location following a peripheral cue was stronger in the temporal than in the nasal hemifield.

Mulckhuyse and Theeuwes (2010) found that briefly presented (16 ms) cues influenced attentional orienting, even when they were not consciously perceived. Specifically, they observed inhibition of return for cues presented in the temporal hemifield but this was not the case for cues presented to the nasal hemifield. Again, this argues that temporal hemifield stimuli (that project to the nasal hemiretina) have a stronger influence on attentional orienting than nasal hemifield stimuli. Similarly, 
Ansorge (2003) showed that masked primes had a stronger effect on performance when they were presented in the temporal than the nasal hemifield.

Berger and Henik (2000) measured the influence of both endogenous precues (such as an arrow at centre pointing to a peripheral location) and exogenous precues (appearing at the target location) on the inhibition of return (IOR) effect. When they tested this influence monocularly, endogenous cues to the nasal hemifield reduced IOR while no such modulation of IOR was observed for cues to the temporal visual hemifield where the effects of the two cue types were independent. This suggested that attentional capture from an exogenous precue is stronger towards the temporal than the nasal hemifield, again consistent with the proposal that stimuli projecting to the nasal hemiretina induce a stronger effect upon attention.

NTAs in attention have also been found with electroencephalography (EEG). Huber-Huber et al. (2015) found higher amplitudes of the N2pc (second negative deflection, that appears typically around $200 \mathrm{~ms}$ after the appearance of the stimulus; pc stands for posterior-contralateral) for targets presented in the nasal than the temporal visual hemifield while an irrelevant distractor was presented in the other hemifield. They concluded that this somewhat unexpected NTA in N2pc reflected stronger inihition of distractors in the temporal than the nasal hemifield. Recent evidence (Li et al. 2018) suggests, however, that $\mathrm{N} 2 \mathrm{pc}$ reflects target enhancement rather than distractor inhibition. The NTA in N2pc found by Huber-Huber et al. (2015) could therefore also reflect stronger target enhancement in the temporal than the nasal hemifield.

It has been shown that facial emotion can affect visual search (Kristjánsson et al. 2013) and eye movements (Bannerman et al. 2010). Kristjánsson et al. found that response times following fearful faces were longer than following neutral faces. In the saccade task, Bannerman et al. used fearful or neutral faces as valid or invalid cues. When the cue was a fearful face and valid, the latency was longer than when the cue was a neutral face. However, this was reversed when the cue was invalid. Framorando et al. (2017) studied NTAs in the EEG signal depending on whether faces had fearful or neutral expressions. The faces were always presented in pairs to the left and right of fixation, either one neutral and one fearful or two neutral faces. When the faces disappeared, a target (" $n$ " or " $m$ ") was presented either at the location of the fearful or 
neutral face. The task was to judge whether the target was an "m" or " $n$ " by pushing the corresponding button. Behaviourally, there were no differences between conditions. But fearful faces in the temporal hemifield elicited larger N2pc amplitudes than faces in the nasal hemifield. Targets elicited greater P1 responses (140-170 ms after target presentation) in the nasal than temporal hemifield but no difference was found in N1 (210-240 ms after target appearance). Framorando et al. (2017) suggested that the larger P1 for nasal hemifield than temporal hemifield targets might reflect stronger inhibition of neutral faces in the temporal than nasal hemifield.

Overall, the evidence from attentional tasks shows that the neurophysiological asymmetries between the nasal and temporal hemifields reviewed above do not only cause performance asymmetries in basic visual tasks, but also in the function of visual attention.

\subsection{Nasal temporal asymmetries in saccades}

There is a general consensus in the literature that visual attention and saccadic eye movements are strongly interdependent, while the strength and nature of this relationship is still hotly debated (Deubel \& Schneider 1996; Hoffman \& Subramaniam 1995; Kowler et al. 1995; Kristjánsson et al. 2001; Rizzolatti, 1987; see Kristjánsson, 2011 for review). Findings on attentional asymmetries have therefore led to a number of studies of NTAs in the characteristics of saccadic eye movements.

A question that has received considerable interest is whether anatomical asymmetries correspond to asymmetries in saccadic latency. In spite of many studies on this topic, the answer cannot at present be considered unequivocal in either direction.

Kristjánsson et al. (2004, experiment 1) tested observers in a step paradigm on a reflexive prosaccade and voluntary antisaccade task (antisaccades involve eye movements in the opposite direction to a presented target), while observers had one eye closed with an eye patch. They found an interesting interaction where the latencies of prosaccades towards stimuli in the temporal hemifield were lower than for saccades into the nasal hemifield. Antisaccades made in response to stimuli in the temporal hemifield were also slower than antisaccades made in response to nasal hemifield targets. In the antisaccade task, observers had to make an eye movement in the opposite 
direction to where the target appeared and if the target was in the temporal hemifield it seemed to have a stronger pull effect than nasal hemifield targets. This result is very interesting since it suggests that NTAs in saccade latency are more related to the location of the visual stimulus than to the saccade direction. Indeed, if the NTA would be related to saccade direction, it should have reversed during the antisaccade task with shorter saccade latencies when the saccade (and not the visual stimulus) was directed toward the temporal hemifield. This result of faster prosaccades into the temporal hemifield has, however, not always been replicated (Bompas et al., 2008; Honda, 2002; Jóhannesson et al. 2012).

In a comprehensive test involving a number of different experiments, Jóhannesson et al. (2012) studied potential NTAs in latency under various conditions, testing different amplitudes, and different amounts of concurrent attentional load. They found no NTAs in latency in any of their testing conditions, and only a minor effect on landing point accuracy. They speculated that cortical input to eye control centres in the mesencephalon may mitigate any asymmetries in visual pathways.

In a recent paper, however, Jóhannesson et al. (2018) investigated training effects during express saccade generation. Their experiments were inspired by the work of Bibi and Edelman (2009) who found that express saccade proportions increased with training when participants made saccades in the gap paradigm. The logic of Jóhannesson et al. (2018) went as follows: Perhaps any saccade latency differences by hemifield are masked by differences in saccade training between individuals, and measuring potential NTAs at different levels of training may actually mask the NTAs. They trained their participants over 12 training sessions of 280 trials in the gap paradigm while participants wore an eye patch over their non-dominant eye. Overall, the proportion of express saccades increased, but the most interesting finding was how asymmetries in both express saccade proportion and latency developed over training. The training increased the proportion of express saccades (latency between 75 and 130 $\mathrm{ms}$ ) and reduced saccadic latency, overall. Initially, the participants made more express saccades into the nasal visual hemifield than into the temporal visual hemifield but after the training this was reversed. Although Jóhannesson et al. (2018) did not find significant differences in latency between hemifields overall, the interaction between 
visual field and training was significant. Before training, latencies were higher towards the temporal than nasal visual hemifields but with training this reversed.

Koller and Rafal (2018) recently assessed NTAs in express saccade latency. Their study involved the gap paradigm with monocular viewing. They separately analysed saccades with latencies between 70 and $150 \mathrm{~ms}$ (considered express saccades), and saccades with latencies between 150 and $350 \mathrm{~ms}$ (considered regular saccades). Their analyses revealed that NTAs in saccade latency occurred only for express saccades; not regular saccades. Together, the findings of Jóhannesson et al. (2018) and of Koller and Rafal (2018) suggest that there are considerable NTAs in latency for express saccades. This contrasts with the small, or non-existent NTAs for regular saccades (Jóhannesson et al., 2012; Tagu et al., 2018a).

If NTAs in saccade latency are restricted to express saccades, this raises the question of why an attentional benefit does not always translate into a benefit for regular saccades. Express saccades differ from regular saccades in that they are assumed to primarily involve the retinotectal pathway (Dorris et al. 1997; Edelman \& Keller 1996, 1998). It is therefore possible that the NTAs for express saccades are driven by retinotectal NTAs. However, as mentioned above, the thalamocortical pathway also shows anatomical and functional NTAs (Connolly \& Van Essen, 1984; Williams et al., 1995; Toosy et al., 2001; Tyschen \& Burkhatler, 1997). The absence of NTAs in the latencies of regular saccades therefore still remains to be explained. We speculate that for the thalamocortical pathway, the saccade generation network receives a lot of topdown influence from other cortical areas (Pierrot-Deseilligny et al., 1995) which are known to induce strong modulations in saccade latency (Sommer \& Wurtz, 2000; Leigh $\&$ Zee, 2006). Cortical input to mesencephalic saccade control centres may therefore dilute any saccadic NTAs. These top-down influences in the generation of regular saccades may compete with the NTAs of the visual system and therefore prevent them from appearing in the latency of regular saccades (see also Bompas et al., 2008 and Tagu et al., 2018a for similar arguments). Another possibility is that attentional NTAs only cause speeded attentional selection but not speeded saccade execution. The attentional benefit may simply not translate into a quicker saccade response (as argued by Jóhannesson et al., 2012). 
In addition to latency, a common measure of saccade performance involves assessing their peak velocity. In contrast to saccade latency, saccadic peak velocity has been shown to involve robust NTAs, with higher peak velocity for temporal than for nasal saccades. This initial finding of Hyde (1959) has been replicated many times with different methods (Collewijn et al. 1988; Cook et al. 1966; Fricker, 1971; Jóhannesson \& Kristjánsson, 2013; Robinson, 1964; Tagu et al., 2018a). For example, using monocular recordings of eye movements under monocular viewing conditions, Jóhannesson and Kristjánsson (2013) measured peak velocities as a function of hemifield, by performing a reanalysis of the data from Jóhannesson et al. (2012). Their results revealed that saccadic peak velocities were higher for saccades towards temporal than nasal hemifield targets. Tagu et al. (2018a) using binocular recordings of eye movements also found strong NTAs in saccadic peak velocity. Interestingly, this NTA was found for both reflexive and voluntary saccades elicited in step and overlap paradigms. Jóhannesson et al. (2018) showed that the NTA in peak velocity was also found for express saccades in the gap paradigm. On the one hand, this result is in line with the neurophysiological asymmetries, that the denser neural circuitry in the nasal hemiretina would lead to higher peak velocity into the temporal visual hemifield. But, on the other hand, this was surprising as it had been assumed for a while that a constant ratio (the main sequence) determined the relationship between velocity and amplitude (Bahill et al. 1975). There was no corresponding increase in amplitude in the data that could have explained this.

The intriguing question is why there are strong and robust NTAs in saccadic peak velocity while such NTAs are not consistently found in saccade latency. One explanation could be that, in contrast to saccadic latency, peak velocity is not affected by top-down influences such as decision making or visual attention (Galley, 1989; see also Di Stasi et al., 2013; Leigh \& Zee, 2006). For saccade latency, the NTAs may therefore be present but not observable because of top-down influences, which mask the NTAs. Importantly, the fact that NTAs in saccadic peak velocity are found whatever the saccade type, reinforces the idea that NTAs are present in all the visual pathways; in the retinotectal pathway which contributes to the generation of express saccades, and in the thalamocortical pathway which is involved in reflexive and voluntary saccade generation. 


\section{Other asymmetries in the visual system}

Although this review has mainly focused on NTAs, the visual system presents many asymmetries. One asymmetry that has raised interest is pseudoneglect (Bowers \& Heilman, 1980; for review, see Jewell \& McCourt, 2000). Pseudoneglect refers to the leftward error typically found in healthy individuals during line bisection tasks, and has been proposed to be related to the specialization of the right cerebral hemisphere for visuo-spatial attention (e.g., Toba, Cavanagh \& Bartolomeo, 2011; Zago et al., 2017). As pseudoneglect is a well-documented phenomenon and because several literature reviews have already been devoted to it (e.g., Jewell \& McCourt, 2000; Ribolsi et al., 2015), we will not discuss it further in this section. We will however discuss other visual asymmetries, first in Section 3.1 between abducting and adducting eye movements (i.e., studies of NTAs during binocular viewing). Then, vertical asymmetries in visual and attentional functions are discussed in Section 3.2, followed by a discussion of centripetal and centrifugal saccades in Section 3.3.

\subsection{Asymmetries found in binocular studies}

In the studies reviewed above on saccadic eye movements, NTAs were assessed with monocular recording during monocular viewing, by contrasting leftward and rightward saccades from the same eye. However, by using binocular recordings of eye movements in more ecologically valid viewing conditions, it is possible to assess NTAs by contrasting the saccadic parameters of the left and right eyes for the saccades generated by each eye at the same time. This method, that eliminates the effects of biological NTAs by combining the nasal and temporal hemifields, has revealed that the two eyes are not always strictly conjugated, and that saccades of the abducting eye have shorter latency and higher peak velocity than concomitant saccades of the adducting eye (Miyoshi et al., 1981; Collewijn et al. 1988). It is important to note that adducting and abducting eye movements can be from one visual field to the other (see Figure 2) and can therefore be affected by the properties of nasal and temporal eye movements as well as properties of centripetal and centrifugal saccades.

Collewijn et al. (1988) performed a very detailed investigation of a large number of saccadic parameters during such binocular recordings. They showed, using scleral coil binocular eye tracking, that saccades made by the abducting eye (the one moving 
away from the anterior-posterior centre line of the body) consistently had shorter duration and higher peak velocity than the concomitant saccade of the adducting eye (the one moving towards the anterior-posterior centre line of the body). Tagu et al. (2018a) systematically investigated NTAs in saccade latency, amplitude, peak velocity and duration with infrared video-based binocular eye tracking. Moreover, their study involved a large variety of horizontal saccades triggered by step and overlap paradigms, and centripetal and centrifugal saccades, made from different starting positions on the horizontal axis. They showed that asymmetries in peak velocity between saccades made simultaneously by the left and right eyes were very robust for all saccade types tested. For a given saccade, the abducting eye did indeed move faster than the adducting eye. However, Tagu et al. (2018a) did not find any NTAs or abducting-adducting differences in saccade latency, amplitude nor duration for any saccade type. Again, this shows that saccade asymmetries are more clearly found in saccadic peak velocity than other parameters which are more sensitive to other top-down influences (Di Stasi et al., 2013; Galley et al., 1989; Leigh \& Zee, 2006).

All in all, by either using monocular recordings of eye movements in monocular viewing conditions (Jóhannesson et al., 2012, 2013; Kristjánsson et al., 2004) or binocular recordings of eye movements in binocular viewing conditions (Miyoshi et al. 1981; Collewijn et al., 1988; Tagu et al., 2018a), research has shown that biological NTAs, found in the retina and the optic nerve, affect saccadic eye movements, leading to clear NTAs in peak velocity. Moreover, these NTAs can also affect the saccade latency of express saccades triggered by the gap paradigm (Jóhannesson et al., 2018; Koller \& Rafal, 2018); although they are not as easily observable for reflexive and voluntary saccades triggered by the step and overlap paradigms, respectively (Jóhannesson et al., 2012; Tagu et al., 2018a). Interestingly express saccades are the eye movement that is probably the least affected by top-down influences (see Section 2.5).

\subsection{Vertical asymmetries in the visual system}

Until now, we have reviewed asymmetries found between the temporal and nasal hemiretinae and abducting and adducting eye movements. However, asymmetries between the upper and lower visual hemifields, or between the processing of visual 
information presented straight-ahead (see section 3.3) and presented elsewhere in the periphery have also been found.

Curcio and colleagues (Curcio \& Allen, 1990; Curcio et al., 1987) showed not only that the density of cones and ganglion cells was higher in the nasal than temporal hemiretina as discussed previously, but also that it was higher in the upper than in the lower hemiretina. As is the case for NTAs, this asymmetry between the upper hemiretina (lower visual hemifield) and the lower hemiretina (upper visual hemifield) seems to spread across the thalamocortical pathway. Indeed, visual objects have been shown to be predominantly represented by neural mechanisms corresponding to the lower visual hemifield in the LGN (Schein \& de Monasterio, 1987) and in V1 (Van Essen et al. 1984). Importantly, the asymmetry continues to spread further into extrastriate visual areas (Rossit et al., 2013; Van Essen et al. 1986) and influence the well-known dorsal stream of vision for action, and ventral stream of vision for perception (Goodale \& Milner, 1992; Ungerleider \& Mishkin, 1982). The lower visual hemifield may have stronger connections with the dorsal stream of vision for action whereas the upper visual hemifield is more connected to the ventral stream of vision for perception (Galletti et al. 1999; Gamberini et al., 2011; Previc, 1990; Rossit et al., 2013). In a recent study, Zhou et al. (2017) proposed that this asymmetry was related to peripersonal and extrapersonal spaces. Accordingly, visually-guided actions initiated by the dorsal stream are mainly executed in peripersonal space; which often occurs in the lower visual hemifield. In contrast, the extrapersonal visual environment is mostly perceived in the upper visual hemifield and would mainly involve object recognition or other perceptual processes. Several psychophysical experiments have indeed shown better performance for visually-guided actions in the lower visual hemifield than in the upper one (Amenedo et al. 2007; Genzano et al. 2001; Rubin et al. 1996; Thomas \& Elias, 2011). Moreover, discrimination performance in egocentric reference frames has been shown to be enhanced in the lower visual hemifield whereas it was enhanced in the upper visual hemifield when the task was performed within an allocentric reference frame (Levine, \& McAnany, 2005; Zhou et al., 2017).

Vertical asymmetries in saccadic latency has been found. Abegg et al. (2015) they presented targets at $45^{\circ}, 135^{\circ}, 225^{\circ}$ and $325^{\circ}$ from the horizontal midline at $10^{\circ}$ eccentricity. Latencies were significantly shorter for saccades into the upper, than the 
lower hemifield. Previously, Zhou and King (2002) had shown that saccades made by macaque monkeys (Macaca mulatta) had shorter latency and higher peak velocity when they were directed toward the upper hemifield than the lower one.

Carrasco et al. (2001) showed that contrast sensitivity was higher for the lower than the upper half of the vertical meridian - a so-called vertical meridian asymmetry. Talgar and Carrasco (2002) then concluded that this was due to visual rather than attentional factors. But asymmetries between the upper and lower visual hemifields have since been found (e.g. Kristjánsson \& Sigurdardottir, 2008; see also Rezec \& Dobkins, 2004) where the attentional boost from precues was higher for the upper than the lower hemifield. This was only true for a disk displacement discrimination task but not Gabororientation as tested by Talgar and Carrasco, however. Kristjánsson and Sigurdardóttir speculated that this asymmetrical effect of attention reflected that because of the lower density of receptors devoted to the upper visual hemifield this part requires a larger attentional boost for stimulus detection (see also Breitmeyer et al., 1975; Carrasco et al., 2004b; for converging results).

\subsection{Centripetal and centrifugal asymmetries}

Another well-known visual asymmetry concerns the perceptual advantage of visual information presented straight-ahead compared to information presented elsewhere (Durand et al. 2012; Kardamakis \& Moschovakis, 2009; Tatler, 2007). This has been linked to privileged processing in V1 of information presented straight-ahead, both in monkeys (Durand et al. 2010; Przybyszewski et al. 2014) and humans (Strappini et al., 2015). This advantage has been proposed to be behaviorally driven, allowing quick orientation to stimuli presented straight-ahead which could be obstacles during locomotion (Durand et al., 2010; Camors et al., 2016). Moreover, the asymmetry between the straight-ahead direction and other positions in the visual field influences saccadic parameters, as centripetal saccades (directed toward the straight-ahead direction) have been shown to have shorter latency (Krebs et al., 2010, 2012), shorter duration (Camors et al., 2016; Collewijn et al., 1988; Pelisson \& Prablanc, 1988) and higher peak velocity (Abel et al. 1979; Camors et al., 2016; Collewijn et al., 1988; Frost \& Pöppel, 1976; Inchingolo et al. 1987; Jürgens et al. 1981; Pelisson \& Prablanc, 1988; Tagu et al., 2018a) than centrifugal saccades (directed away from the straight-ahead). Importantly, Tagu et al. (2018a) showed that this centripetal-centrifugal asymmetry 
(CCA) in saccadic parameters was found for temporal saccades only, not nasal saccades. This finding suggests that NTA and CCA share some links. The CCA has been proposed to be related to asymmetrical mechanical constraints at the muscular level on the lateral- and medial extraocular rectus (Koene \& Erkelens, 2002). However, other studies have shown that the CCA also had subcortical and cortical correlates. Paré and Munoz (2001) found that the preparatory activity of the buildup neurons in the SC was higher for centripetal, than for centrifugal saccades (in the monkey, using single cell recording) while Krebs et al. (2010; in humans using fMRI) found the activity in the SC to be lower for centripetal, than for centrifugal saccades. In Paré and Munoz's study the centripetal-centrifugal direction did neither affect the activity of the fixation nor the burst neurons which, along with different methods, might explain the seeming contradiction between those studies. In an EEG study, Krebs et al. (2012) found that parieto-occipital regions contralateral to the saccade direction showed a negative deflection $160 \mathrm{~ms}$ before saccade onset. Importantly, this deflection was shorter and weaker prior to centripetal than centrifugal saccades. Krebs et al. (2012) proposed that the weaker deflection suggest a lower need for attentional guidance for centripetal than for centrifugal saccades.

When the eye is in its primary position, the lateral and medial recti are mostly relaxed (Sparks, 2002) and the mechanical forces of the socket can keep the eye stable in this position. As soon as the eye is to be rotated, one rectus must be contracted while the other is kept in its relaxed condition. However, the elastic force of the relaxing rectus increases, the further the eye is rotated away from its primary position. After the eye has been rotated away from its primary position the force of the pulling rectus is adjusted sufficiently to overcome the elastic force of the relaxed rectus and to keep the eyeball at the new position (Sparks, 2002). When the eye rotates back to its primary position the relaxing of the pulling rectus should suffice to bring the eye back to its primary position. But some force of the previously relaxed rectus may be added to the rotation to speed up the rotation process which can increase the peak velocity of centripetal saccades over centrifugal saccades (Abel et al. 1979; Camors et al., 2016; Collewijn et al., 1988; Frost \& Pöppel, 1976; Inchingolo et al. 1987; Jürgens et al. 1981; Pelisson \& Prablanc, 1988; Tagu et al., 2018a). This could also contribute to the shorter latency of centripetal than of centrifugal saccades (Krebs et al., 2010, 2012) 


\section{Individual variability in NTAs}

While NTAs in saccadic eye movement parameters have been found in several studies from different laboratories using different eye-tracking techniques, it is important to highlight at this point that a subgroup of participants do not show any NTAs.

Vergilino-Perez et al. (2012) investigated potential left-right asymmetries in saccadic latency, amplitude and peak velocity of saccades under binocular viewing (during binocular viewing biological NTAs are eliminated, see Section 2), with both monocular and binocular recording. They did not find any asymmetries in latency and only a weak NTA in saccade amplitude. However, their most interesting result was that during binocular recordings of eye movements, some participants showed robust NTAs in peak velocity but others did not. Instead, these participants showed either higher peak velocities for leftward than rightward saccades, or for rightward than leftward saccades in both eyes; irrespective of whether the saccades were temporal or nasal. Moreover, the participants with higher leftward than rightward peak velocities had a dominant left eye while those showing higher peak velocities rightward than leftward had a dominant right eye. In other words, for these participants peak velocities were consistently higher for saccades into the hemifield ipsilateral to the dominant eye. Vergilino-Perez et al. (2012) therefore proposed that the peak velocity pattern was linked to eye dominance and that these participants could have stronger eye dominance than those exhibiting standard NTAs where peak velocities were not linked to eye dominance. Interestingly, this shows that studies of NTAs in saccadic parameters could reveal individual characteristics such as the strength of eye dominance. Moreover, other studies contrasting participants with "strong" eye dominance (without any NTA in saccadic peak velocity) against participants with "weak" eye dominance (exhibiting standard NTA in saccadic peak velocity) showed that this measure of eye dominance strength based on oculomotor behavior influenced perceptual (Chaumillon et al., 2017) and visuo-motor (Tagu et al. 2016) performance.

In Tagu et al. (2016) participants with left- or right eye dominance made saccades toward a target presented along with a proximal distractor. This situation is known to induce a "global effect" (Findlay, 1982; Vitu, 2008; Walker et al., 1997): the saccade landing position falls in between the saccade target and the distractor. Tagu et al. (2016) measured this global effect separately for the left- and right visual hemifields. 
Participants with strong eye dominance had higher saccade accuracy (weaker global effect) in the hemifield contralateral to the dominant eye than in the ipsilateral hemifield. This contralateral advantage was not found in participants with weak eye dominance (exhibiting standard NTAs). These results reinforce the idea that NTAs can be used to quantify eye dominance strength. Two questions remain however: why the hemifield contralateral to the dominant eye has such advantages, and why NTAs are linked to eye dominance strength.

It is well known that similar factors can influence eye dominance and the ocular dominance columns in the visual cortex, such as lack of visual input as shown in deprivation studies (LeVay et al. 1980; Shatz \& Stryker, 1978). Neuroimaging studies have shown that V1 ipsilateral to the dominant eye is larger (Erdogan et al., 2002) and more highly activated when the dominant eye was stimulated than when the nondominant eye was stimulated (Shima et al., 2010). This suggests that there is a particular relationship between the dominant eye and ipsilateral V1. Due to the architecture of the thalamocortical pathway, V1 ipsilateral to the dominant eye processes information presented in the contralateral hemifield. Tagu et al. (2016) proposed that the higher saccade accuracy found in the hemifield contralateral to the dominant eye reflected this. Moreover, this only occurs for participants with strong eye dominance (no NTA in saccadic peak velocity), so the relationship between the dominant eye and the ipsilateral V1 should be present in this population only. Interestingly, using magnetoencephalography, Shima et al. (2010) found that V1 showed higher activation when the dominant eye was stimulated (by presenting a reversing checkerboard stimulus) than when the non-dominant eye was stimulated; but only when the stimulus was presented in the nasal hemifield. This suggests that the relationship between the dominant eye and ipsilateral V1 is strongly related to input from the temporal hemiretina. We believe that this could be the reason why NTAs and eye dominance strength are linked. Interestingly, V1 ocular dominance columns receiving input from the nasal hemiretina are larger and occupy more area than the ones receiving input from the temporal hemiretina (Tychsen \& Burkhalter, 1997), while ocular dominance columns in V1 ipsilateral to the dominant eye (input from temporal hemiretina) seem more activated than the ones receiving input from the nasal hemiretina (Shima et al., 2010). Competition between these two phenomena may therefore attenuate, or even eliminate, behavioral NTAs. This would suggest that the stronger the relationship 
between the dominant eye and ipsilateral V1 (i.e., the higher the eye dominance strength), the lower the observed NTA.

Interestingly, as for NTAs, other asymmetries found in the visual system also show individual variability (e.g., Camors et al., 2016; Galley, 1989; Tagu et al., 2018a). We therefore believe that these asymmetries could be linked to individual characteristics such as eye dominance. Indeed, Tagu et al. (2018b) showed that simultaneously studying individual variabilities in NTA and CCA provided a graded measure of eye dominance, reinforcing the idea that NTA and CCA share some links. We however acknowledge that biological evidence regarding this question is sparse. Future studies should be carried out to further understand this individual variability found in the asymmetries of the visual system, and how it may relate to the neuroanatomy and physiology of the visual system.

\section{Conclusions and future directions}

The evidence that we have reviewed clearly shows that there are various forms of asymmetries in the neurophysiological mechanisms supporting visual perception and oculomotor mechanisms. The evidence also shows that all these asymmetries found in the visual system influence visual performance, attentional function and eye movements. Most surprising, perhaps, is that these asymmetries seem to interact. One recommendation that we can therefore make is that these asymmetries must be taken into account in current and future research in visual attention, visual perception, oculomotor control and eye movements before experimental results are interpreted. Additionally, during eye tracking studies, attention must be paid to which eye is measured - the left or right, dominant or non-dominant - (which is not always reported), since performance can clearly differ by which eye is measured, and the saccade direction (for example for studies involving only one saccade direction). Finally, studies of asymmetries of the visual system can clearly inform our understanding of the visual system, and can in many cases lead to surprising insights into visual perception, oculomotor behavior and the neural mechanisms involved.

Although this review has provided new insights into visual asymmetries, it also has highlighted unresolved issues, that future studies may address. Firstly, the study of NTAs in saccade latency suggest that they are found only for express, not regular 
saccades. This discrepancy is important as it directly concerns the neurophysiology of saccadic asymmetries, and could for example be driven by different expression of NTAs in the retinotectal and thalamocortical pathways. The differences between NTAs in express and regular saccades may be examined in future studies involving both behavioral and neurophysiological measures. Furthermore, the particular emphasis of the NTAs in express saccades and retinotectal projections in different species could provide an evolutionary perspective of the NTAs, as this pathway is shared by almost all vertebrates. Although we reviewed evidence regarding the development of NTAs, none of these studies included investigations of the development of saccadic NTAs. Yet, the huge advances in eye-tracking technologies made over the last decades now allow examining the saccadic eye movements of preschool children with high temporal and spatial accuracy (e.g., Alahyane et al., 2016; Lemoine-Lardennois et al., 2016). Future studies are needed to examine how the NTAs in saccadic parameters evolve during development. This point could also give new insights about the links between saccadic asymmetries and eye dominance. Indeed, although evidence is sparse, it seems that eye dominance is not fully established until after three years of age. Infants often switch between the two eyes to perform monocular tasks (Coren et al. 1981; Updegraff, 1932). As the saccadic NTAs in peak velocity seem mostly observed in case of weak eye dominance, they may be enhanced in preschool infants, and decrease after three years of age when eye dominance is established.

Future studies concerning these questions may furthermore provide new information about the links between the different saccadic asymmetries (e.g., Tagu et al., 2018a) and between saccadic asymmetries and eye dominance (e.g., Tagu et al., 2018b).

A final puzzle that we are left with concerns two proposals that have been reviewed regarding asymmetrical processing; firstly, the point that nasal-temporal asymmetries in visual and attentional processing may reflect the need to quickly orient to events in the periphery, and secondly that the centripetal-centrifugal asymmetry reflects a bias towards potentially important items straight ahead in the visual field. We consider it a highly interesting endeavor to address this seeming contradiction. But keep in mind, that there may not be contradiction since the somewhat haphazard nature of evolution leaves open the possibility that two independent mechanisms may have 
developed in parallel or at different evolutionary stages serving these two different functions.

Author contributions: OIJ made the figures and took care of the reference list. Otherwise the authors contributed equally to all parts of the paper.

Conflict of interest: The authors declare that there is no conflict of interest regarding this paper.

\section{Abbreviations}

CCA: centripetal-centrifugal asymmetry.

EEG: electroencephalography.

ERG: electroretinogram.

IOR: inhibition of return.

LGN: Lateral geniculate nucleus.

N2pc: The second negative deflection in the EEG spectrum and appears typically around $200 \mathrm{~ms}$ after the appearance of the stimulus; pc stands for posteriorcontralateral.

NTA: Nasal-temporal asymmetry.

P1: The first positive component in the EEG spectrum and appears typically $100 \mathrm{~ms}$ after the appearance of the stimulus.

SC: Superior colliculus.

V1: The primary visual area of the cerebral cortex 


\section{References}

Abegg, M., Pianezzi, D., \& Barton, J. J. S. (2015). A Vertical Asymmetry in Saccades. Journal of Eye Movement Research, 8(5). http://doi.org/10.16910/jemr.8.5.3

Abel, L. A., Dell'Osso, L. F., Daroff, R. B., \& Parker, L. (1979). Saccades in extremes of lateral gaze. Investigative Ophthalmology \& Visual Science, 18(3), 324-327.

Alahyane, N., Lemoine-Lardennois, C., Tailhefer, C., Collins, T., Fagard, J., \& DoréMazars, K. (2016). Development and learning of saccadic eye movements in 7-to 42-month-old children. Journal of vision, 16(1), 6-6. doi:10.1167/16.1.6

Amenedo, E., Pazo-Alvarez, P., \& Cadaveira, F. (2007). Vertical asymmetries in preattentive detection of changes in motion direction. International Journal of Psychophysiology, 64(2), 184-189. https://doi.org/10.1016/j.ijpsycho.2007.02.001

Anderson, R. S., Wilkinson, M. O., \& Thibos, L. N. (1992). Psychophysical localization of the human visual streak. Optometry \& Vision Science, 69(3), 171-174. http://doi.org/10.1097/00006324-199203000-00001

Anderson, S. J., Mullen, K. T., \& Hess, R. F. (1991). Human peripheral spatial resolution for achromatic and chromatic stimuli: limits imposed by optical and retinal factors. The Journal of Physiology, 442(1), 47-64. http://doi.org/10.1113/jphysiol.1991.sp018781

Ansorge, U. (2003). Asymmetric influences of temporally vs. nasally presented masked visual information: Evidence for collicular contributions to nonconscious priming effects* 1 . Brain and Cognition, 51(3), 317-325.

Anstis, S. M. (1974). A chart demonstrating variations in acuity with retinal position. Vision Research, 14(7), 589-592. http://doi.org/10.1016/0042-6989(74)90049-2

Bahill, A. T., Clark, M. R., \& Stark, L. (1975). The main sequence, a tool for studying human eye movements. Mathematical Biosciences, 24(3-4), 191-204.

Bannerman, R. L., Milders, M., \& Sahraie, A. (2010). Attentional bias to brief threatrelated faces revealed by saccadic eye movements. Emotion, 10(5), 733-738. http://doi.org/10.1037/a0019354

Becker, W. (1989). The neurobiology of saccadic eye movements. Metrics. In R. H. Wurtz \& M. E. Goldberg (Eds.), Reviews of Oculomotor Research, 3, (pp. 13-66). Michigan: Elsevier.

Berger, A., \& Henik, A. (2000). The endogenous modulation of IOR is nasal-temporal asymmetric. Journal of Cognitive Neuroscience, 12(3), 421-428.

Bibi, R., \& Edelman, J. A. (2009). The influence of motor training on human express saccade production. Journal of Neurophysiology. http://doi.org/10.1152/jn.90710.2008

Bompas, A., \& Sumner, P. (2008). Sensory sluggishness dissociates saccadic, manual, and perceptual responses: An S-cone study. Journal of Vision, 8(8), 1-13. http://doi.org/10.1167/8.8.10

Bompas, A., Sterling, T., Rafal, R. D., \& Sumner, P. (2008). Naso-temporal asymmetry for signals invisible to the retinotectal pathway. Journal of Neurophysiology, 100(1), $412-421$. 
Bowering, E. R., Maurer, D., Lewis, T. L., \& Brent, H. P. (1993). Sensitivity in the nasal and temporal hemifields in children treated for cataract, Investigative Ophthalmology \& Visual Science, 34(13), 3501-3509.

Bowers, D. \& Heilman, K. M. (1980). Pseudoneglect : Effects of hemispace on a tactile line bisection task. Neuropsychologia, 18(4-5), 491-498.

Breitmeyer, B., Julesz, B., \& Kropfl, W. (1975). Dynamic random-dot stereograms reveal up-down anisotropy and left-right isotropy between cortical hemifields. Science, 187(4173), 269-270.

Camors, D., Trotter, Y., Pouget, P., Gilardeau, S., \& Durand, J.-B. (2016). Visual straight-ahead preference in saccadic eye movements. Scientific Reports, 6:23124, 1-9. http://doi.org/10.1038/srep23124

Carrasco, M., Ling, S., \& Read, S. (2004a). Attention alters appearance. Nature Neuroscience, 7(3), 308-313. http://doi.org/10.1038/nn1194

Carrasco, M., Marie Giordano, A., \& McElree, B. (2004b). Temporal performance fields: visual and attentional factors. Vision Research, 44(12), 1351-1365. http://doi.org/10.1016/j.visres.2003.11.026

Carrasco, M., Talgar, C. P., \& Cameron, E. L. (2001). Characterizing visual performance fields: Effects of transient covert attention, spatial frequency, eccentricity, task and set size. Spatial vision, 15(1), 61-75.

Chaumillon, R., Alahyane, N., Senot, P., Vergne, J., Lemoine-Lardennois, C., Blouin, J., Doré-Mazars, K., Guillaume, A., \& Vergilino-Perez, D. (2017). Asymmetry in visual information processing depends on the strength of eye dominance. Neuropsychologia, 96 , 129-136. http://doi.org/10.1016/j.neuropsychologia.2017.01.015

Collewijn, H., Erkelens, C. J., \& Steinman, R. M. (1988). Binocular co-ordination of human horizontal saccadic eye movements. The Journal of Physiology, 404, 157182.

Connolly, M., \& Van Essen, D. (1984). The representation of the visual field in parvicellular and magnocellular layers of the lateral geniculate nucleus in the macaque monkey. The Journal of Comparative Neurology, 226(4), 544-564. https://doi.org/10.1002/cne.902260408

Cook, G., Stark, L., \& Zuber, B. L. (1966). Horizontal eye movements studied with the on-line computer. Archives of Ophthalmology (Chicago, Ill.: 1960), 76(4), 589-595.

Coren, S., Porac, C., \& Duncan, P. (1981). Lateral preference behaviors in preschool children and young adults. Child Development, 52(2), 443-450.

Curcio, C., \& Allen, K. (1990). Topography of ganglion cells in human retina. The Journal of Comparative Neurology, 300(1), 5-25.

Curcio, C. A., Sloan, K. R., Packer, O., Hendrickson, A. E., \& Kalina, R. E. (1987). Distribution of cones in human and monkey retina: individual variability and radial asymmetry. Science, 236(4801), 579-582. http://doi.org/10.1126/science.3576186

Delinte, A., Gomez, C., Decostre, M., Crommelinck, M., \& Roucoux, A. (2002). Amplitude transition function of human express saccades. Neuroscience, 42, 21-34. 
Deubel, H., \& Schneider, W. (1996). Saccade target selection and object recognition: Evidence for a common attentional mechanism. Vision Research, 36(12), 18271837.

Di Stasi, L. L., Catena, A., Canas, J. J., Macknik, S. L., \& Martinez-Conde, S. (2013). Saccadic velocity as an arousal index in naturalistic tasks. Neuroscience \& Biobehavioral Reviews, 37(5), 968-975. https://doi.org/10.1016/j.neubiorev.2013.03.011

Dodds, C., Machado, L., Rafal, R., \& Ro, T. (2002). A temporal/nasal asymmetry for blindsight in a localisation task: evidence for extrageniculate mediation. NeuroReport, 13(5), 655.

Dorris, M. C., Paré, M., \& Munoz, D. P. (1997). Neuronal activity in monkey superior colliculus related to the initiation of saccadic eye movements. Journal of Neuroscience, 17(21), 8566-8579.

Durand, J.-B., Camors, D., Trotter, Y., \& Celebrini, S. (2012). Privileged visual processing of the straight-ahead direction in humans. Journal of Vision, 12(6):34, 113. http://doi.org/10.1167/12.6.34

Durand, J.-B., Trotter, Y., \& Celebrini, S. (2010). Privileged processing of the straightahead direction in primate area V1. Neuron, 66(1), 126-137.

Edelman, J. A., \& Keller, E. L. (1998). Dependence on target configuration of express saccade-related activity in the primate superior colliculus. Journal of Neurophysiology, 80, 1407-1426.

Edelman, J., \& Keller, E. (1996). Activity of visuomotor burst neurons in the superior colliculus accompanying express saccades. Journal of Neurophysiology, 76(2), 908 926.

Edelman, J. A., Kristjansson, A., \& Nakayama, K. (2007). The influence of objectrelative visuomotor set on express saccades. Journal of Vision, 7(6), 12-12. http://doi.org/10.1167/7.6.12

Erdogan, A. R., Özdikici, M., Aydin, M. D., Aktas, Ö., \& Dane, S. (2002). Right and left visual cortex areas in healthy subjects with right-and left-eye dominance. International Journal of Neuroscience, 112(5), 517-523.

Fahle, M. (1987). Naso-temporal asymmetry of binocular inhibition. Investigative Opthalmology \& Visual Science, 28(6), 1016-1017.

Fahle, M., \& Schmid, M. (1988). Naso-temporal asymmetry of visual perception and of the visual cortex. Vision Research, 28(2), 293-300.

Findlay, J. M. (1982). Global visual processing for saccadic eye movements. Vision Research, 22(8), 1033-1045. https://doi.org/10.1016/0042-6989(82)90040-2

Fischer, B., \& Boch, R. (1983). Saccadic eye movements after extremely short reaction times in the monkey. Brain research, 260(1), 21-26.

Fischer, B., \& Ramsperger, E. (1984). Human express saccades: extremely short reaction times of goal directed eye movements. Experimental Brain Research, 57(1), 191-195.

Fischer, B., \& Weber, H. (1993). Express saccades and visual attention. Behavioral and Brain Sciences, 16(03), 553. http://doi.org/10.1017/s0140525x00031575 
Framorando, D., Bapst, M., Vuille, N., \& Pegna, A. J. (2017). Naso-temporal asymmetries: Suppression of emotional faces in the temporal visual hemifield. Frontiers in Neuroscience, 11, 1635-8. http://doi.org/10.3389/fnins.2017.00014

Fricker, S. J. (1971). Dynamic measurements of horizontal eye motion I. Acceleration and velocity matrices. Investigative Ophthalmology \& Visual Science, 10(9), 724732 .

Frost, D., \& Pöppel, E. (1976). Different programming modes of human saccadic eye movements as a function of stimulus eccentricity: Indications of a functional subdivision of the visual field. Biological Cybernetics, 23(1), 39-48. http://doi.org/10.1007/BF00344150.

Galletti, C., Fattori, P., Kutz, D. F., \& Gamberini, M. (1999). Brain location and visual topography of cortical area V6A in the macaque monkey. European Journal of Neuroscience, 11(2), 575-582. https://doi.org/10.1046/j.1460-9568.1999.00817.x

Galley, N. (1989). Saccadic eye movement velocity as an indicator of (de) activation: A review and some speculations. Journal of Psychophysiology, 3, 229-244.

Gamberini, M., Galletti, C., Bosco, A., Breveglieri, R., \& Fattori, P. (2011). Is the medial posterior parietal area V6A a single functional area?. Journal of Neuroscience, 31(13), 5145-5157. https://doi.org/10.1523/JNEUROSCI.548910.2011

Genzano, V. R., Di Nocera, F., \& Ferlazzo, F. (2001). Upper/lower visual field asymmetry on a spatial relocation memory task. NeuroReport, 12(6), 1227-1230.

Goodale, M. A., \& Milner, A. D. (1992). Separate visual pathways for perception and action. Trends in Neurosciences, 15(1), 20-25. http://doi.org/10.1016/01662236(92)90344-8

Grigsby, S. S., \& Tsou, B. H. (1994). Grating and flicker sensitivity in the near and far periphery: Naso-temporal asymmetries and binocular summation. Vision Research, 34(21), 2841-2848. http://doi.org/10.1016/0042-6989(94)90053-1

Hall, W.C., \& Lee, P. (1997). Interlaminar connections of the superior colliculus in the tree shrew. III: the optic layer. Vis. Neurosci. 14(4), 647-661. http://dx.doi.org/10.1017/S095252380001261X.

Hoffman, J., \& Subramaniam, B. (1995). The role of visual attention in saccadic eye movements. Perception and Psychophysics, 57(6), 787-795.

Heeman, J., Van der Stigchel, S., \& Theeuwes, J. (2017). The influence of distractors on express saccades. Journal of Vision, 17(1), 35-17. http://doi.org/10.1167/17.1.35

Hoffman, J., \& Subramaniam, B. (1995). The role of visual attention in saccadic eye movements. Perception and Psychophysics, 57(6), 787-795.

Honda, H. (2002). Idiosyncratic left-right asymmetries of saccadic latencies: examination in a gap paradigm. Vision Research, 42(11), 1437-1445.

Hubel, D. H., LeVay, S., \& Wiesel, T. N. (1975). Mode of termination of retinotectal fibers in macaque monkey: an autoradiographic study. Brain Research, 96(1), 2540 .

Huber-Huber, C., Grubert, A., Ansorge, U., \& Eimer, M. (2015). Nasotemporal ERP differences: evidence for increased inhibition of temporal distractors. Journal of Neurophysiology, 113(7), 2210-2219. http://doi.org/10.1152/jn.00344.2014 
Inchingolo, P., Spanio, M., \& Bianchi, M. (1987). The characteristic peak velocityMean velocity of saccadic eye movements in man. In J.K. O'Regan \& A. LévySchoen (Eds.), Eye Movements from Physiology to Cognition: Selected/Edited Proceedings of the Third European Conference on Eye Movements, Dourdan, France (pp. 17-26). North-Holland: Elsevier.

Itaya, S. K., \& Van Hoesen, G. W. (1983). Retinal projections to the inferior and medial pulvinar nuclei in the old-world monkey. Brain Research, 269(2), 223-230.

Jewell, G. \& McCourt, M. E. (2000). Pseudoneglect : a review and meta-analysis of performance factors in line bisection tasks. Neuropsychologia, 38(1), 93-110

Johnson, M. H. (2005). Subcortical face processing. Nature Reviews Neuroscience, 6, 766-774. http://doi.org/10.1038/nrnl766

Johnson, M. H., Farroni, T., Brockbank, M., \& Simion, F. (2000). Preferential orienting to faces in 4-month-olds: analysis of temporal-nasal visual field differences. Developmental Science, 3(1), 41-45. http://doi.org/10.1111/1467-7687.00097

Jóhannesson, Ó. I., Ásgeirsson, Á. G., \& Kristjánsson, Á. (2012). Saccade performance in the nasal and temporal hemifields. Experimental Brain Research, 219(1), 107120. http://doi.org/10.1007/s00221-012-3071-2

Jóhannesson, Ó. I., Edelman, J. A., Sigurpórsson, B. D., \& Kristjánsson, Á. (2018). Effects of saccade training on express saccade proportions, saccade latencies, and peak velocities: an investigation of nasal/temporal differences. Experimental Brain Research, 84(3-4), 1. http://doi.org/10.1007/s00221-018-5213-7

Jóhannesson, Ó. I., \& Kristjánsson, Á. (2013). Violating the main sequence: asymmetries in saccadic peak velocities for saccades into the temporal versus nasal hemifields. Experimental Brain Research, 227(1), 101-110. http://doi.org/10.1007/s00221-013-3490-8

Jürgens, R., Becker, W., \& Kornhuber, H. H. (1981). Natural and drug-induced variations of velocity and duration of human saccadic eye movements: Evidence for a control of the neural pulse generator by local feedback. Biological Cybernetics, 39(2), 87-96. http://doi.org/10.1007/BF00336734

Kardamakis, A. A., \& Moschovakis, A. K. (2009). Optimal control of gaze shifts. $\begin{array}{llll}\text { Journal of } & \text { Neuroscience, } & \text { 7723-7730. }\end{array}$ http://doi.org/10.1523/JNEUROSCI.5518-08.2009

Keltgen, K. M., \& Swanson, W. H. (2012). Estimation of spatial scale across the visual field using sinusoidal stimuli. Investigative Opthalmology \& Visual Science, 53(2), 633-7. http://doi.org/10.1167/iovs.10-6674

Kingstone, A., \& Klein, R. M. (1993). Visual offsets facilitate saccadic latency: Does predisengagement of visuospatial attention mediate this gap effect? Journal of Experimental Psychology: Human Perception and Performance, 19(6), 1251-1265. http://doi.org/10.1037/0096-1523.19.6.1251

Knox, P. C., \& Wolohan, F. D. A. (2015). Temporal stability and the effects of training on saccade latency in "Express Saccade Makers." PLoS ONE, 10(3), e0120437-16. http://doi.org/10.1371/journal.pone.0120437 
Knox, P. C., Wolohan, F. D. A., \& Helmy, M. S. (2017). Express saccades in distinct populations: east, west, and in-between. Experimental Brain Research, 1-11. http://doi.org/10.1007/s00221-017-5094-1

Koene, A. R., \& Erkelens, C. J. (2002). Cause of kinematic differences during centrifugal and centripetal saccades. Vision Research, 42(14), 1797-1808.

Koller, K., \& Rafal, R. D. (2018). Saccade latency bias toward temporal hemifield: evidence for role of retinotectal tract in mediating reflexive saccades. Neuropsychologia. https://doi.org/10.1016/j.neuropsychologia.2018.01.028

Kowler, E., Anderson, E., Dosher, B., \& Blaser, E. (1995). The role of attention in the programming of saccades. Vision Research, 35(13), 1897-1916.

Knudsen, E. I. (2011). Control from below: the role of a midbrain network in spatial attention. European Journal of Neuroscience, 33(11), 1961-1972.

Krauzlis, R. J., Lovejoy, L. P., \& Zénon, A. (2013). Superior colliculus and visual spatial attention. Annual Review of Neuroscience, 36(1), 165-182. http://doi.org/10.1146/annurev-neuro-062012-170249

Krebs, R. M., Boehler, C. N., Zhang, H. H., Schoenfeld, M. A., \& Woldorff, M. G. (2012). Electrophysiological recordings in humans reveal reduced location-specific attentional-shift activity prior to recentering saccades. Journal of Neurophysiology, 107(5), 1393-1402. http://doi.org/10.1152/jn.00912.2010

Krebs, R. M., Schoenfeld, M. A., Boehler, C. N., Song, A. W., \& Woldorff, M. G. (2010). The saccadic recentering bias is associated with activity changes in the human superior colliculus. Frontiers in Human Neuroscience, 4:193, 1-12. http://doi.org/10.3389/fnhum.2010.00193

Kristjánsson, Á. (2011). The intriguing interactive relationship between visual attention and saccadic eye movements. In L. Liversedge, I. D. Gilchrist, \& S. Everling, Oxford Handbook of Eye Movements (1st ed., Vol. 1, pp. 455-470). Oxford: Oxford University Press.

Kristjansson, A., Chen, Y., \& Nakayama, K. (2001). Less attention is more in the preparation of antisaccades, but not prosaccades. Nature Neuroscience, 4, 10371042.

Kristjansson, A., \& Nakayama, K. (2003). A primitive memory system for the deployment of transient attention. Perception and Psychophysics, 65(5), 711-724.

Kristjánsson, Á., Óladóttir, B., \& Most, S. B. (2013). "Hot" facilitation of 'cool' processing: Emotional distraction can enhance priming of visual search. Journal of Experimental Psychology: Human Perception and Performance, 39(1), 298-306. http://doi.org/10.1037/a0028683

Kristjánsson, Á., \& Sigurdardottir, H. M. (2008). On the benefits of transient attention across the visual field. Perception, 37(5), 747-764. http://doi.org/10.1068/p5922

Kristjansson, A., Vandenbroucke, M., \& Driver, J. (2004). When pros become cons for anti-versus prosaccades: factors with opposite or common effects on different saccade types. Experimental Brain Research, 155(2), 231-244.

Leigh, R. J., \& Zee, D. S. (2006). The saccadic system. In R. J. Leigh \& D. S. Zee (Eds.), The neurology of eye movements, 4th edition (pp.108-187). New York: Oxford University Press. 
Lemoine-Lardennois, C., Alahyane, N., Tailhefer, C., Collins, T., Fagard, J., \& DoréMazars, K. (2016). Saccadic Adaptation in 10-41 Month-Old Children. Frontiers in human neuroscience, 10, 241. doi:10.3389/fnhum.2016.00241

LeVay, S., Connolly, M., Houde, J., \& Van Essen, D. C. (1985). The complete pattern of ocular dominance stripes in the striate cortex and visual field of the macaque monkey. The Journal of Neuroscience, 5(2), 486-501.

LeVay, S., Wiesel, T. N., \& Hubel, D. H. (1980). The development of ocular dominance columns in normal and visually deprived monkeys. Journal of Comparative Neurology, 191(1), 1-51.

Levine, M. W., \& McAnany, J. J. (2005). The relative capabilities of the upper and lower visual hemifields. Vision Research, 45(21), 2820-2830. http://doi.org/10.1016/j.visres.2005.04.001

Lewis, T. L., \& Maurer, D. (1992). The development of the temporal and nasal visual fields during infancy. Vision Research, 32(5), 903-911.

Lewis, T. L., Maurer, D., \& Blackburn, K. (1985). The development of young infants' ability to detect stimuli in the nasal visual field. Vision Research, 25(7), 943-950.

Lévy-Schoen, A. (1969). Détermination et latence de la réponse oculomotrice à deux stimulus simultanés ou successifs selon leur excentricité relative. L'année Psychologique, 69(2), 373-392.

Li, C., Liu, Q., \& Hu, Z. (2018). Further evidence that N2pc reflects target enhancement rather than distracter suppression. Frontiers in Psychology, 8:2275, 1-6. https://doi.org/10.3389/fpsyg.2017.02275

Miyoshi, T., Hiwatashi, S., Kishimoto, S., \& Tamada, A. (1981). Dissociation of the eyes in saccadic movement. Annals of the New York Academy of Sciences, 374(1), 731-743. http://doi.org/10.1111/j.1749-6632.1981.tb30914.x

Mulckhuyse, M., \& Theeuwes, J. (2010). Unconscious cueing effects in saccadic eye movements - Facilitation and inhibition in temporal and nasal hemifield. Vision Research, 50(6), 606-613. http://doi.org/10.1016/j.visres.2010.01.005

Osterberg, G. (1935). Topography of the layer of rods and cones in the human retina. Copenhagen: Nyt nordisk forlag.

Paradiso, M. A., \& Carney, T. (1988). Orientation discrimination as a function of stimulus eccentricity and size: Nasal/temporal retinal asymmetry. Vision Research, 28(8), 867-874. http://doi.org/10.1016/0042-6989(88)90096-х

Paré, M., \& Munoz, D. P. (2001). Expression of a recentering bias in saccade regulation by superior colliculus neurons. Experimental Brain Research, 137, 354-368.

Pelisson, D., \& Prablanc, C. (1988). Kinematics of centrifugal and centripetal saccadic eye movements in man. Vision Research, 28(1), 87-94. http://doi.org/10.1016/S0042-6989(88)80009-9

Perry, V. H., \& Cowey, A. (1985). The ganglion cell and cone distributions in the monkey's retina: Implications for central magnification factors. Vision Research, 25(12), 1795-1810. http://doi.org/10.1016/0042-6989(85)90004-5

Perry, V. H., Oehler, R., \& Cowey, A. (1984). Retinal ganglion cells that project to the dorsal lateral geniculate nucleus in the macaque monkey. Neuroscience, 12(4), 1101-1123. 
Pierrot - Deseilligny, C., Rivaud, S., Gaymard, B., Müri, R., \& Vermersch, A. I. (1995). Cortical control of saccades. Annals of neurology, 37(5), 557-567.

Posner, M., \& Cohen, Y. (1980). Attention and the control of movements. Tutorials in Motor Behavior, 243-258.

Previc, F. H. (1990). Functional specialization in the lower and upper visual fields in humans: Its ecological origins and neurophysiological implications. Behavioral and Brain Sciences, 13(3), 519-542. https://doi.org/10.1017/S0140525X00080018

Przybyszewski, A. W., Kagan, I., \& Snodderly, D. M. (2014). Primate area V1: Largest response gain for receptive fields in the straight-ahead direction. NeuroReport, 25(14), 1109-1115.

Rachel, R. and Reese, B. (2009). Optic nerve, optic chiasm, and optic tracts. In. LR, Squire (Ed.), Encyclopedia of Neuroscience (pp. 249-254). Oxford: Academia Press.

Rafal, R. D., Calabresi, P. A., Brennan, C. W., \& Sciolto, T. K. (1989). Saccade preparation inhibits reorienting to recently attended locations. Journal of Experimental Psychology: Human Perception and Performance, 15(4), 673-685. http://doi.org/10.1037//0096-1523.15.4.673

Rafal, R. D., Henik, A., \& Smith, J. (1991). Extrageniculate contributions to reflex visual orienting in normal humans: A temporal hemifield advantage. Journal of Cognitive Neuroscience, 3(4), 322-328.

Rafal, R., Smith, J., Krantz, A., Cohen, A., \& Brennan, C. (1990). Extrageniculate vision in hemianopic humans: Saccade inhibition by signals in the blind field. Science, 250, 118-121.

Rezec, A., \& Dobkins, K. (2004). Attentional weighting: A possible account of visual field asymmetries in visual search? Spatial Vision, 17(4), 269-293. http://doi.org/10.1163/1568568041920203

Ribolsi, M., Di Lorenzo, G., Lisi, G., Niolu, C., \& Siracusano, A. (2015). A critical review and meta-analysis of the perceptual pseudoneglect across psychiatric disorders: Is there a continuum?. Cognitive Processing, 16(1), 17-25.

Rizzolatti, G., Riggio, L., Dascola, I., \& Umiltá, C. (1987). Reorienting attention across the horizontal and vertical meridians: evidence in favor of a premotor theory of attention. Neuropsychologia, 25(1), 31-40.

Robinson, D. A. (1964). The mechanics of human saccadic eye movement. The Journal of physiology, 174(2), 245-264.

Rolls, E. T., \& Cowey, A. (1970). Topography of the retina and striate cortex and its relationship to visual acuity in rhesus monkeys and squirrel monkeys. Experimental Brain Research, 10(3), 298-310. http://doi.org/10.1007/BF00235053

Rossit, S., McAdam, T., McLean, D. A., Goodale, M. A., \& Culham, J. C. (2013). fMRI reveals a lower visual field preference for hand actions in human superior parietooccipital cortex (SPOC) and precuneus. Cortex, 49(9), 2525-2541. https://doi.org/10.1016/j.cortex.2012.12.014

Rovamo, J., \& Virsu, V. (1979). An estimation and application of the human cortical magnification factor. Experimental Brain Research, 37(3), 495-510. http://doi.org/10.1007/BF00236819 
Rothbart, M. K., Posner, M. I., \& Boylan, A. (1990). Chapter 3 Regulatory Mechanisms in Infant Development. In The Development of attention - Research and Theory (Vol. 69, pp. 47-66). Elsevier. http://doi.org/10.1016/S0166-4115(08)60450-1

Rubin, N., Nakayama, K., \& Shapley, R. (1996). Enhanced perception of illusory contours in the lower versus upper visual hemifields. Science, 271(5249), 651-653. http://doi.org/10.1126/science.271.5249.651

Saslow, M. G. (1967). Effects of components of displacement-step stimuli upon latency for saccadic eye movement. Josa, 57(8), 1024-1029. http://doi.org/10.1364/JOSA.57.001024

Schein, S. J., \& de Monasterio, F. M. (1987). Mapping of retinal and geniculate neurons onto striate cortex of macaque. Journal of Neuroscience, 7(4), 996-1009. https://doi.org/10.1523/JNEUROSCI.07-04-00996.1987

Shatz, C. J., \& Stryker, M. P. (1978). Ocular dominance in layer IV of the cat's visual cortex and the effects of monocular deprivation. The Journal of Physiology, 281(1), 267-283.

Shima, H., Hasegawa, M., Tachibana, O., Nomura, M., Yamashita, J., Ozaki, Y., Kawai, J., Higuchi, M., \& Kado, H. (2010). Ocular dominance affects magnitude of dipole moment: an MEG study: NeuroReport, 21(12), 817 - 821. http://doi.org/10.1097/WNR.0b013e32833ce5d7

Silva, M. F., Brascamp, J. W., Ferreira, S., Castelo-Branco, M., Dumoulin, S. O., \& Ben M Harvey. (2018). Radial asymmetries in population receptive field size and cortical magnification factor in early visual cortex. NeuroImage, 167, 41-52. http://doi.org/10.1016/j.neuroimage.2017.11.021

Silva, M. F., Maia-Lopes, S., Mateus, C., Guerreiro, M., Sampaio, J., Faria, P., \& Castelo-Branco, M. (2008). Retinal and cortical patterns of spatial anisotropy in contrast sensitivity tasks. Vision Research, 48(1), 127-135. http://doi.org/10.1016/j.visres.2007.10.018

Simion, F., Valenza, E., Umiltá, C., \& Barba, B. D. (1995). Inhibition of return in newborns is temporo-nasal asymmetrical. Infant Behavior and Development, 18(2), 189-194. http://doi.org/10.1016/0163-6383(95)90048-9

Simion, F., Valenza, E., Umiltá, C., \& Barba, B. D. (1998). Preferential orienting to faces in newborns: A temporal-nasal asymmetry. Journal of Experimental Psychology: Human Perception and Performance, 24(5), 1399-1405. http://doi.org/10.1037/0096-1523.24.5.1399

Sommer, M. A., \& Wurtz, R. H. (2000). Composition and topographic organization of signals sent from the frontal eye field to the superior colliculus. Journal of Neurophysiology, 83(4), 1979-2001.

Sparks, D. L. (2002). The brainstem control of saccadic eye movements. Nature Reviews Neuroscience, 3, 952-964.

Sylvester, R., Josephs, O., Driver, J., \& Rees, G. (2007). Visual fMRI responses in human superior colliculus show a temporal-nasal asymmetry that is absent in lateral geniculate and visual cortex. Journal of Neurophysiology, 97(2), 1495-1502. http://doi.org/10.1152/jn.00835.2006 
Sterling, P. (1973). Quantitative mapping with the electron microscope: Retinal terminals in the superior colliculus. Brain Research, 54, 347- 354.

Strappini, F., Pitzalis, S., Snyder, A. Z., McAvoy, M. P., Sereno, M. I., Corbetta, M., \& Shulman, G. L. (2015). Eye position modulates retinotopic responses in early visual areas: A bias for the straight-ahead direction. Brain Structure and Function, 220(5), 2587-2601.

Sutter, E. E., \& Tran, D. (1992). The field topography of ERG components in man-I. The photopic luminance response. Vision Research, 32(3), 433-446. http://doi.org/10.1016/0042-6989(92)90235-b

Tagu, J., Doré-Mazars, K., Lemoine-Lardennois, C., \& Vergilino-Perez, D. (2016). How eye dominance strength modulates the influence of a distractor on saccade accuracy. Investigative Ophthalmology \& Visual Science, 57(2), 534-543. http://doi.org/10.1167/iovs.15-18428

Tagu, J., Doré-Mazars, K., Vergne, J., Lemoine-Lardennois, C., \& Vergilino-Perez, D. (2018a). Recentering bias for temporal saccades only: Evidence from binocular recordings of eye movements. Journal of Vision, 18(1):10, 1-16, https://doi.org/10.1167/18.1.10

Tagu, J., Doré-Mazars, K., Vergne, J., Lemoine-Lardennois, C., \& Vergilino-Perez, D. (2018b). Quantifying eye dominance strength - New insights into the neurophysiological bases of saccadic asymmetries. Neuropsychologia, 117, 530540, https://doi.org/10.1016/j.neuropsychologia.2018.07.020

Talgar, C. P., \& Carrasco, M. (2002). Vertical meridian asymmetry in spatial resolution: Visual and attentional factors. Psychonomic Bulletin \& Review, 9(4), 714-722.

Tatler, B. W. (2007). The central fixation bias in scene viewing: Selecting an optimal viewing position independently of motor biases and image feature distributions. Journal of Vision, 7(14):4, 1-17, http://doi.org/10.1167/7.14.4.

Tigges, J., \& Tigges, M. (1981). Distribution of retionfugal and corticofugal axon terminals in the superior colliculus of squirrel monkey. Investigative Ophthalmology and Visual Science, 20, 149- 158.

Thibos, L. N., Cheney, F. E., \& Walsh, D. J. (1987). Retinal limits to the detection and resolution of gratings. JOSA A, 4(8), 1524-1529.

Thomas, N. A., \& Elias, L. J. (2011). Upper and lower visual field differences in perceptual asymmetries. Brain Research, 1387, 108-115. https://doi.org/10.1016/j.brainres.2011.02.063

Thorpe, S., Fize, D., \& Marlot, C. (1996). Speed of processing in the human visual system. Nature, 381(6582), 520-522. http://doi.org/10.1038/381520a0

Toba, M.-N., Cavanagh, P., \& Bartolomeo, P. (2011). Attention biases the perceived midpoint of horizontal lines. Neuropsychologia, 49(2), 238-246.

Tomalski, P., Johnson, M. H., \& Csibra, G. (2009). Temporal-nasal asymmetry of rapid orienting to face-like stimuli. NeuroReport, 20(15), 1309-1312. http://doi.org/10.1097/WNR.0b013e32832f0acd 
Toosy, A. T., Werring, D. J., Plant, G. T., Bullmore, E. T., Miller, D. H., \& Thompson, A. J. (2001). Asymmetrical activation of human visual cortex demonstrated by functional MRI with monocular stimulation. Neuroimage, 14(3), 632-641.

Tychsen, L., \& Burkhalter, A. (1997). Nasotemporal asymmetries in V1: Ocular dominance columns of infant, adult, and strabismic macaque monkeys. The Journal of Comparative Neurology, 388(1), 32-46.

Ungerleider L, Mishkin M. (1982). Two cortical visual systems. In D. J. Ingle, M. A. Goodale \& R. J. W. Mansfield (Eds.), Analysis of Motor Behavior (pp. 549-586). Cambridge: MIT Press.

Updegraff, R. (1932). Ocular dominance in young children. Journal of Experimental Psychology, 15(6), 758-766.

Van Essen, D. C., Newsome, W. T., \& Maunsell, J. H. (1984). The visual field representation in striate cortex of the macaque monkey: asymmetries, anisotropies, and individual variability. Vision Research, 24(5), 429-448. https://doi.org/10.1016/0042-6989(84)90041-5

Van Essen, D. C., Newsome, W. T., Maunsell, J. H. R., \& Bixby, J. L. (1986). The projections from striate cortex (V1) to areas V2 and V3 in the macaque monkey: asymmetries, areal boundaries, and patchy connections. Journal of Comparative Neurology, 244(4), 451-480. https://doi.org/10.1002/cne.902440405

Vergilino-Perez, D., Fayel, A., Lemoine, C., Senot, P., Vergne, J., \& Doré-Mazars, K. (2012). Are there any left-right asymmetries in saccade parameters? Examination of latency, gain, and peak velocity. Investigative Ophthalmology \& Visual Science, 53(7), 3340-3348. http://doi.org/ 10.1167/iovs.11-9273

Vitu, F. (2008). About the global effect and the critical role of retinal eccentricity: Implications for eye movements in reading. Journal of Eye Movement Research, 2(3). http://doi.org/10.16910/jemr.2.3.6

Vuilleumier, P., \& Schwartz, S. (2001). Emotional facial expressions capture attention. Neurology, 56(2), 153-158. http://doi.org/10.1212/WNL.56.2.153

Walker, R., Mannan, S., Maurer, D., Pambakian, A. L. M., \& Kennard, C. (2000b). The oculomotor distractor effect in normal and hemianopic vision. Proceedings of the Royal Society of London B: Biological Sciences, 267(1442), 431-438. http://doi.org/10.1098/rspb.2000.1018

Walker, R., Walker, D. G., Husain, M., \& Kennard, C. (2000a). Control of voluntary and reflexive saccades. Experimental Brain Research, 130(4), 540-544. http://doi.org/10.1007/s002219900285

Walker, R., Deubel, H., Schneider, W., \& Findlay, J. (1997). Effect of remote distractors on saccade programming: evidence for an extended fixation zone. Journal of Neurophysiology, 78(2), 1108-1119-1119.

Wässle, H., Grünert, U., Röhrenbeck, J., \& Boycott, B. B. (1990). Retinal ganglion cell density and cortical magnification factor in the primate. Vision research, 30(11), 1897-1911.

Wässle, H., Grünert, U., Röhrenbeck, J., \& Boycott, B. B. (1989). Cortical magnification factor and the ganglion cell density of the primate retina. Nature, 341(6243), 643. 
Wenban-Smith, M. G., \& Findlay, J. M. (1991). Express saccades: is there a separate population in humans? Experimental Brain Research, 87(1), 218-222. http://doi.org/10.1007/BF00228523

Weiskrantz, L. (1996). Blindsight revisited. Current Opinion in Neurobiology, 6(2), 215-220. http://doi.org/10.1016/s0959-4388(96)80075-4

White, B. J., \& Munoz, D. P. (2011). The superior colliculus. In S. P. Liversedge, I. D. Gilchrist, \& S. Everling (Eds.), The Oxford handbook of eye movements (pp.195213). New York: Oxford University Press.

Williams, C., Azzopardi, P., \& Cowey, A. (1995). Nasal and temporal retinal ganglion cells projecting to the midbrain: implications for "blindsight." Neuroscience, 65(2), 577-586.

Whitteridge, D. (1965). Geometrical relations between the retina and the visual cortex. In Mathematics and Computer Science in Biology and Medicine (pp. 269-276). Medical Research Council London.

Zago, L., Petit, L., Jobard, G., Hay, J., Mazoyer, B., Tzourio-Mazoyer, N., Karnath, H.O., \& Mellet, E. (2017). Pseudoneglect in line bisection judgement is associated with a modulation of right hemispheric spatial attention dominance in right-handers. Neuropsychologia, 94, 75-83.

Zhou, W., \& King, W. M. (2002). Attentional sensitivity and asymmetries of vertical saccade generation in monkey. Vision Research, 42(6), 771-779. http://doi.org/10.1016/s0042-6989(01)00319-4

Zhou, Y., Yu, G., Yu, X., Wu, S., \& Zhang, M. (2017). Asymmetric representations of upper and lower visual fields in egocentric and allocentric references. Journal of Vision, 17(1):9, 1-11, http://doi.org/10.1167/17.1.9 